\title{
FIRST ORDER $k$-TH MOMENT FINITE ELEMENT ANALYSIS OF NONLINEAR OPERATOR EQUATIONS WITH STOCHASTIC DATA
}

\begin{abstract}
ALEXEY CHERNOV AND CHRISTOPH SCHWAB
Dedicated to W. L. Wendland on the occasion of his 75th anniversary

Abstract. We develop and analyze a class of efficient Galerkin approximation methods for uncertainty quantification of nonlinear operator equations. The algorithms are based on sparse Galerkin discretizations of tensorized linearizations at nominal parameters. Specifically, we consider abstract, nonlinear, parametric operator equations $J(\alpha, u)=0$ for random input $\alpha(\omega)$ with almost sure realizations in a neighborhood of a nominal input parameter $\alpha_{0}$. Under some structural assumptions on the parameter dependence, we prove existence and uniqueness of a random solution, $u(\omega)=S(\alpha(\omega))$.

We derive a multilinear, tensorized operator equation for the deterministic computation of $k$-th order statistical moments of the random solution's fluctuations $u(\omega)-S\left(\alpha_{0}\right)$. We introduce and analyse sparse tensor Galerkin discretization schemes for the efficient, deterministic computation of the $k$-th statistical moment equation. We prove a shift theorem for the $k$-point correlation equation in anisotropic smoothness scales and deduce that sparse tensor Galerkin discretizations of this equation converge in accuracy vs. complexity which equals, up to logarithmic terms, that of the Galerkin discretization of a single instance of the mean field problem. We illustrate the abstract theory for nonstationary diffusion problems in random domains.
\end{abstract}

\section{INTRODUCTION}

We consider uncertainty quantification for abstract, parametric, nonlinear problems: given $\alpha \in Z$, find $u \in X$ such that

$$
J(\alpha, u)=0 \quad \text { in } Y^{\prime} .
$$

Here $X, Y, Z$ are Banach spaces, $Y^{\prime}$ is the dual of $Y, u \in X$ is the unknown solution and $\alpha \in Z$ represents the set of inputs (such as boundary conditions, operator coefficients, shape of the physical domain of $u$, etc.). We allow the functional

$$
J:\left\{\begin{aligned}
Z \times X & \rightarrow Y^{\prime} \\
(\alpha, u) & \mapsto J(\alpha, u)
\end{aligned}\right.
$$

Received by the editor August 12, 2011 and, in revised form, February 5, 2012.

2010 Mathematics Subject Classification. Primary 65N30, 65J15.

Key words and phrases. Nonlinear operator equations, random parameters, deterministic methods, Fréchet derivative, sparse tensor approximation, random domain.

This work was supported in part under grant ERC AdG 247277 (to CS), initiated at the Institute for Mathematics and Applications (IMA) in Minnesota, USA and completed at the Hausdorff Research Institute for Mathematics, Bonn, Germany during the trimester "High dimensional problems" May-August 2011. The first author acknowledges support by the Hausdorff Center for Mathematics, Bonn and the second author acknowledges the excellent working conditions at the Hausdorff Research Institute. 
to depend nonlinearly on both variables, but insist on it to satisfy structural assumptions which render it locally, in a vicinity of a nominal parameter value $\alpha_{0} \in Z$, well-posed (these structural assumptions on (1.1) will be made precise later); there exists a unique, "nominal" solution $u_{0} \in X$ such that $J\left(\alpha_{0}, u_{0}\right)=0$ in $Y^{\prime}$.

Well-posedness of problem (1.1) implies continuous dependence of solutions on data: changes in the problem data in some (usually sufficiently small) open neighborhood $U \subset Z$ of the nominal parameter value $\alpha_{0} \in U$ lead to a corresponding small change of $u_{0} \in X$ such that (1.1) is satisfied. We denote the solution operator of (1.1) by $S: U \rightarrow X$,

$$
u=S(\alpha) \quad \text { in } X, \quad \alpha \in U
$$

and assume that $S$ is injective in $U$.

In many practical applications, the effective values of the problem parameters $\alpha$ are not known precisely, but are known (or assumed) to be close to certain nominal values $\alpha_{0}$ so that the fluctuation

$$
r=\alpha-\alpha_{0} \neq 0 .
$$

Uncertainty in the parameter values can be accounted for by probabilistic modelling of the input's fluctuation $r=r(\omega)=\alpha-\alpha_{0}$ as a random field with known law or statistical moments. We show that if $\alpha(\omega)=\alpha_{0}+r(\omega)$ belongs to $U$ almost surely, $u=u(\omega)$ becomes a well-defined random field (cf. Lemma 4.1 below). One goal of this work is to present deterministic strategies towards the efficient, deterministic computation of its statistics. Our approach is based on the (formal, at this stage) first order Taylor expansion of $J$ at the nominal values $\left(\alpha_{0}, u_{0}\right)$ : if $J(\alpha, u)$ is twice Fréchet differentiable at the nominal values $\left(\alpha_{0}, u_{0}\right)$, say, then for all $(\alpha, u)$ in a vicinity of $\left(\alpha_{0}, u_{0}\right)$ holds

$$
\begin{aligned}
J(\alpha, u)= & J\left(\alpha_{0}, u_{0}\right)+J_{\alpha}^{\prime}\left(\alpha_{0}, u_{0}\right)\left(\alpha-\alpha_{0}\right)+J_{u}^{\prime}\left(\alpha_{0}, u_{0}\right)\left(u-u_{0}\right) \\
& +\mathcal{O}\left(\left\|\alpha-\alpha_{0}\right\|_{Z}^{2}\right)+\mathcal{O}\left(\left\|u-u_{0}\right\|_{X}^{2}\right) .
\end{aligned}
$$

Here, the differentials $J_{\alpha}^{\prime}\left(\alpha_{0}, u_{0}\right) \in \mathcal{L}\left(Z, Y^{\prime}\right)$ and $J_{u}^{\prime}\left(\alpha_{0}, u_{0}\right) \in \mathcal{L}\left(X, Y^{\prime}\right)$ are bounded, linear operators which are assumed to be known. Using (1.1) and neglecting the second order remainders yields the first order perturbation equation

$$
0=J_{\alpha}^{\prime}\left(\alpha_{0}, u_{0}\right)\left(\alpha-\alpha_{0}\right)+J_{u}^{\prime}\left(\alpha_{0}, u_{0}\right)\left(u-u_{0}\right) \text { in } Y^{\prime} .
$$

Well-posedness of (1.1) implies that the differential $J_{u}^{\prime}(\alpha, u)$ is boundedly invertible in a vicinity of the nominal pair $\left(\alpha_{0}, u_{0}\right)$. Then

$$
\begin{aligned}
u-u_{0}=S(\alpha)-S\left(\alpha_{0}\right) & =-\left[J_{u}^{\prime}\left(\alpha_{0}, u_{0}\right)\right]^{-1} J_{\alpha}^{\prime}\left(\alpha_{0}, u_{0}\right)\left(\alpha-\alpha_{0}\right)+\mathcal{O}\left(\left\|\alpha-\alpha_{0}\right\|_{Z}^{2}\right) \\
& =S^{\prime}\left(\alpha_{0}\right)\left(\alpha-\alpha_{0}\right)+\mathcal{O}\left(\left\|\alpha-\alpha_{0}\right\|_{Z}^{2}\right) .
\end{aligned}
$$

The aim of this paper is to develop fast, deterministic algorithms for the approximate computation of the $k$-th statistical moment $\mathcal{M}^{k}\left[u-u_{0}\right]$ (also referred to as " $k$ point correlation function") of the random solution $u(\omega)$ from known $k$-th moment of the perturbation $\mathcal{M}^{k} r$. Our algorithms will be based on trading randomness for high dimensionality: rather than sampling (1.1) for numerous realizations of $\alpha$, we derive an abstract, deterministic tensor equation for the $k$-point correlation function of the system's stochastic response: in Theorem 4.8 we prove that $\mathcal{M}^{k}\left[u-u_{0}\right]$ can be approximated by $\mathcal{M}^{k}\left[S^{\prime}\left(\alpha_{0}\right) r\right]$, where $S^{\prime}\left(\alpha_{0}\right) \in \mathcal{L}(Z, X)$ is the Fréchet derivative 
of the solution operator (1.3) at $\alpha_{0}$. One main contribution of the present paper are explicit bounds for the approximation error

$$
\mathcal{E}^{k}=\mathcal{M}^{k}\left[u-u_{0}\right]-\mathcal{M}^{k}\left[S^{\prime}\left(\alpha_{0}\right) r\right] .
$$

The classical Implicit Function Theorem provides existence of a neighborhood $U$ of $\alpha_{0}$ such that $S$ is differentiable in $U$. However, this classical result does not allow us to control the size of $U$, so that we obtain only asymptotic estimates for the approximation error $\mathcal{E}^{k}$ when the size of $U$ tends to zero.

In order to derive explicit nonasymptotic bounds for $\mathcal{E}^{k}$ we prove in the present paper variants of the classical Local Implicit Function Theorem with an explicit relation between the size of $U$ and the size of the differentials of the nonlinear functional $J$. These results are Newton-Kantorovich-type theorems with quantitative control of the constants which may be of independent interest. The local Lipschitz continuity of $J_{u}^{\prime}, J_{\alpha}^{\prime}$ and the local invertibility of $J_{u}^{\prime}$ are the key properties allowing us to control the size of $U$. We provide a detailed regularity analysis for $\mathcal{M}^{k}\left[u-u_{0}\right]$ and $\mathcal{M}^{k}\left[S\left(\alpha_{0}\right) r\right]$.

If the magnitude of $\mathcal{E}^{k}$ is small $\mathbb{P}$-a.s., we propose to compute $\mathcal{M}^{k}\left[S^{\prime}\left(\alpha_{0}\right) r\right]$ instead of $\mathcal{M}^{k}\left[u-u_{0}\right]$, which is a simpler task, since $S^{\prime}\left(\alpha_{0}\right) r$ can be characterized as a solution of a linear operator equation with a random right-hand side. We then apply the tensorization technique [24, 26] to characterize $\mathcal{M}^{k}\left[S^{\prime}\left(\alpha_{0}\right) r\right]$ as the solution of a tensorized deterministic operator equation.

Ultimately, the success of this approach depends, however, on our ability to efficiently solve this tensorized, deterministic operator equation for the $k$-point correlation function. To this end, we extend and generalize the approach of [24] to the present setting by imposing further assumptions on the "sensitivity operator" $G_{0}=J_{u}^{\prime}\left(\alpha_{0}, u_{0}\right)$. We discuss this first in the particular case when $X=Y$ are separable Hilbert spaces and when $G_{0}$ is coercive.

In Section 4, we consider random perturbations of the parameter $\alpha$ in (1.1) which are formalized on a background probability space satisfying the Kolmogorov axioms of probability: throughout, $\mathbb{P}$ shall denote a probability measure on the probability space $(\Omega, \mathcal{A})$. By capitals $X, Y, Z$ we shall denote separable Banach spaces of functions. Solutions to parametric random boundary value problems will be sought in Bochner spaces $L^{p}(\Omega, \mathbb{P} ; X)$ of $p$-summable, measurable mappings from $(\Omega, \mathcal{A})$ to $(X, \mathcal{B}(X))$ where $\mathcal{B}(X)$ denotes the sigma algebra of Borel sets on the separable Banach space $X$.

The paper is organized as follows. We begin with a brief recapitulation of differential calculus in Banach spaces in Section 2. In Section 3 we study properties of the implicit solution of (1.1) with a deterministic parameter $\alpha$. In particular, we obtain explicit bounds on the neighborhood $U \ni \alpha_{0}$ where the solution operator $u=S(\alpha)$ is well defined and is Fréchet differentiable. The size of $U$ depends explicitly on smoothness properties of $J$ and its Fréchet derivatives. In Section 4 we apply these results to (1.1) where $\alpha$ is a random field. In particular, we obtain asymptotic and nonasymptotic bounds on the approximation error $\mathcal{E}^{k}$ from (1.6) in the natural tensor product norms; see Theorem 4.6 and Theorem 4.8, In Section 5 we develop full and sparse tensor product multilevel Galerkin discretization schemes for the abstract $k$-th moment equation. In particular, we obtain a priori convergence estimates under isotropic and mixed regularity assumptions. In Section 6 we illustrate the developed abstract techniques on sparse space-time tensor BEM for nonstationary diffusion problems in random domains. In particular, we propose 
a sparse tensor Galerkin scheme for the approximation of the $k$-point correlation of the random solution at any point in the space-time cylinder with complexity of log-linear order in $N$, the number of degrees of freedom on the nominal boundary.

\section{Differential calculus in Banach spaces}

In this section we recall definitions and results of differential calculus in Banach spaces needed in what follows (see e.g. [2, 4, 17, 6]).

Suppose $X, Y$ and $Z$ are Banach spaces and $U \subset Z$ is an open subset. We denote $C(U, X)$ the space of continuous (w.r.t. convergence in norm) maps $U \rightarrow X$ and by $\mathcal{L}(Z, X)$ the space of linear continuous maps $Z \rightarrow X$.

Definition 2.1. Let $U$ be an open subset of $Z$ and $\alpha \in U$. A mapping $F$ is called Fréchet differentiable at $\alpha$ if there exists a linear continuous mapping $A \in \mathcal{L}(Z, X)$ such that for

$$
R(r)=F(\alpha+r)-F(\alpha)-A(r) \quad \text { there holds }\|R(r)\|_{X}=o\left(\|r\|_{Z}\right),
$$

that is, $\|R(r)\|_{X} /\|r\|_{Z} \rightarrow 0$ as $\|r\|_{Z} \rightarrow 0$. If it exists, $A$ is uniquely defined and is called the Fréchet derivative of $F$ at $\alpha \in Z$ and denoted by $F^{\prime}(\alpha)$. The mapping $F$ is said to be Fréchet differentiable in $U \subset Z$ if $F$ is differentiable at all $\alpha \in U$.

If $F^{\prime}$ is continuous as a map $U \rightarrow \mathcal{L}(Z, X)$ we say that $F$ is $C^{1}$ and we write $F \in C^{1}(U, X)$. Consider a map $J(\cdot, u): Z \rightarrow Y^{\prime}$ for some fixed element $u \in X$. Its differential with respect to $\alpha$ is the Fréchet derivative $J_{\alpha}^{\prime}(\alpha, u) \in \mathcal{L}\left(Z, Y^{\prime}\right)$ at $(\alpha, u)$ with respect to $\alpha$. If it exists, it is uniquely defined by the requirement

$$
\left\|J(\alpha+r, u)-J(\alpha, u)-J_{\alpha}^{\prime}(\alpha, u) r\right\|_{Y^{\prime}}=o\left(\|r\|_{Z}\right)
$$

for all $r \in U$ in a neighborhood of $0 \in U$.

We will also require the following differentiation rule for composite maps.

Lemma 2.2 (Chain rule). Let $F: U \rightarrow X$ be differentiable at $\alpha \in U$ and $G: V \rightarrow$ $Y$ be differentiable at $u=F(\alpha) \in V$, where $U$ and $V$ are open subsets of $Z$ and $X$ respectively. Then $G \circ F: U \rightarrow Y$ is differentiable at $\alpha$ and it holds that

$$
(G \circ F)^{\prime}(\alpha) r=G^{\prime}(u)\left[F^{\prime}(\alpha) r\right], \quad \text { with } u=F(\alpha) \text {. }
$$

Theorem 2.3. Suppose $F \in C^{1}(U, X)$ in an open, nonempty subset $U \subset Z$. Assume that $\alpha \in U$ and $r \in Z$ such that $[\alpha, \alpha+r]:=\{\alpha+\theta r: \theta \in[0,1]\} \subset U$. Then

$$
F(\alpha+r)-F(\alpha)=\int_{0}^{1} F^{\prime}(\alpha+r \theta) r \mathrm{~d} \theta .
$$

The following classical result gives sufficient conditions for local solvability of the equation (1.1); cf. [17, X.§2], [4, Ch. 3.1], [2, Ch. 2].

Theorem 2.4 (Local Implicit Function Theorem). Suppose $X, Y, Z$ are Banach spaces and $W$ is an open neighborhood of $\left(\alpha_{0}, u_{0}\right)$ in $Z \times X$. Suppose $J\left(\alpha_{0}, u_{0}\right)=0$ and

(1) $J_{u}^{\prime}(\alpha, u)$ exists in $W$ and $J, J_{u}^{\prime}$ are continuous in $\left(\alpha_{0}, u_{0}\right)$;

(2) the "sensitivity operator" $G_{0}:=J_{u}^{\prime}\left(\alpha_{0}, u_{0}\right) \in \mathcal{L}\left(X, Y^{\prime}\right)$ admits a bounded inverse $\Gamma_{0} \in \mathcal{L}\left(Y^{\prime}, X\right)$

Then there exist neighborhoods $U$ of $\alpha_{0}$ and $V$ of $u_{0}$ and a unique mapping $S: U \rightarrow$ $V$, such that 
a. $S\left(\alpha_{0}\right)=u_{0}$;

b. $J(\alpha, u)=0$ in $Y^{\prime}$ is equivalent to $u=S(\alpha)$ in $X$ for any $\alpha \in U$, i.e.,

$$
J(\alpha, S(\alpha))=0 \text { holds in } Y^{\prime} \text { for any } \alpha \in U \text {; }
$$

c. $S \in C(U, X)$ if (2) holds and if (1) is replaced with the stronger assumption: (1a) $J \in C\left(W, Y^{\prime}\right), J_{u}^{\prime}(\alpha, u)$ exists in $W$ and $J_{u}^{\prime}$ is continuous in $\left(\alpha_{0}, u_{0}\right)$;

d. if (2) holds and if (1) is replaced with the stronger assumption

(1b) $J_{u}^{\prime}(\alpha, u), J_{\alpha}^{\prime}(\alpha, u)$ exist in $W$ and $J, J_{\alpha}^{\prime}, J_{u}^{\prime}$ are continuous in $\left(\alpha_{0}, u_{0}\right)$, then $S$ is differentiable in $\alpha_{0}$ and it holds that

$$
S^{\prime}\left(\alpha_{0}\right)=-\Gamma_{0} J_{\alpha}^{\prime}\left(\alpha_{0}, u_{0}\right) .
$$

Slightly stronger assumptions on $J$ are needed to ensure Lipschitz continuity of $S$ in $U$. In particular, we have the following theorem.

Theorem 2.5. Suppose the assumptions of Theorem 2.4 are satisfied and assume that $S: U \rightarrow X$ is the solution operator of (1.1). Assume in addition that

(1') $J \in C^{1}\left(W, Y^{\prime}\right)$

(2') the "sensitivity operator" $G_{1}:=J_{u}^{\prime}\left(\alpha_{1}, u_{1}\right) \in \mathcal{L}\left(X, Y^{\prime}\right)$ admits a bounded inverse $\Gamma_{1} \in \mathcal{L}\left(Y^{\prime}, X\right)$ for any $\alpha_{1} \in U$ and $u_{1}=S\left(\alpha_{1}\right)$.

Then $S \in C^{1}(U, X)$ and it holds that

$$
S^{\prime}\left(\alpha_{1}\right)=-\Gamma_{1} J_{\alpha}^{\prime}\left(\alpha_{1}, S\left(\alpha_{1}\right)\right) \quad \forall \alpha_{1} \in U .
$$

In what follows we utilize the following notations for closed and open balls in Banach spaces: for $\alpha \in Z$ and $\delta \geq 0$,

$$
B\left(\alpha_{0}, \delta\right):=\left\{\alpha \in Z:\left\|\alpha-\alpha_{0}\right\|_{Z} \leq \delta\right\}, \quad \stackrel{\circ}{B}\left(\alpha_{0}, \delta\right):=B\left(\alpha_{0}, \delta\right) \backslash \partial B\left(\alpha_{0}, \delta\right) .
$$

In our notation, we do not indicate the Banach space $Z$ explicitly, since it is always clear from the context.

\section{THE SIZE OF NEIGHBORHOODS}

The assumptions of Theorem 2.4 and Theorem 2.5 are not sufficient to control the size of the neighborhood $U$ of around $\alpha_{0}$ where the solution operator $S(\alpha)$ is well defined and is differentiable. Revisiting the classical proofs of this result, for example, in [17, X.§2], 4, Ch. 3.1], allows us to find a direct connection between the size of $U$ and smoothness properties of $J$. The following result shows that the additional assumption of Lipschitz continuity of $J_{\alpha}^{\prime}$ and $J_{u}^{\prime}$ at $\left(\alpha_{0}, u_{0}\right)$ is sufficient for existence of $S(\alpha)$ for $\alpha \in U$ with quantitative bounds on the size of $U$. This result is inspired by the Newton-Kantorovich Theorem in the form stated, for example, in [16, Ch. XVIII], [27, Ch. 5].

Theorem 3.1. Suppose $X, Y, Z$ are Banach spaces and $W$ is an open neighborhood of $\left(\alpha_{0}, u_{0}\right) \in Z \times X$. Suppose $J\left(\alpha_{0}, u_{0}\right)=0$ and that

(1) $J \in C\left(W, Y^{\prime}\right), J_{\alpha}^{\prime}, J_{u}^{\prime}$ exist in $W$ and are Lipschitz continuous in $\left(\alpha_{0}, u_{0}\right)$,

(2) the "sensitivity operator" $G_{0}:=J_{u}^{\prime}\left(\alpha_{0}, u_{0}\right) \in \mathcal{L}\left(X, Y^{\prime}\right)$ has a bounded inverse $\Gamma_{0} \in \mathcal{L}\left(Y^{\prime}, X\right)$.

Define

$$
\xi_{0}:=\left\|\Gamma_{0} J_{\alpha}^{\prime}\left(\alpha_{0}, u_{0}\right)\right\|_{Z \rightarrow X}
$$


and $\eta_{0}$ being the smallest constant satisfying

$$
\left.\begin{array}{l}
\left\|\Gamma_{0}\left\{J_{\alpha}^{\prime}(\alpha, u)-J_{\alpha}^{\prime}\left(\alpha_{0}, u_{0}\right)\right\}\right\|_{Z \rightarrow X} \\
\left\|\Gamma_{0}\left\{J_{u}^{\prime}(\alpha, u)-J_{u}^{\prime}\left(\alpha_{0}, u_{0}\right)\right\}\right\|_{X \rightarrow X}
\end{array}\right\} \leq \eta_{0}\left(\left\|\alpha-\alpha_{0}\right\|_{Z}+\left\|u-u_{0}\right\|_{X}\right)
$$

for any $(\alpha, u) \in W$. Let

$$
\delta_{0}:=\frac{1}{2 \eta_{0}\left(1+\xi_{0}\right)}, \quad \varepsilon_{0}(\delta):=\frac{1-\delta \eta_{0}-\sqrt{1-2 \delta \eta_{0}\left(1+\xi_{0}\right)}}{\eta_{0}}
$$

and assume that $\delta<\delta_{0}$ is so small that $B\left(\alpha_{0}, \delta\right) \times B\left(u_{0}, \varepsilon_{0}(\delta)\right) \subset W$. Then the following holds:

a. There exists a unique continuous mapping $S: B\left(\alpha_{0}, \delta\right) \mapsto B\left(u_{0}, \varepsilon_{0}(\delta)\right)$ satisfying $J(\alpha, S(\alpha))=0$ for any $\alpha \in B\left(\alpha_{0}, \delta\right)$ and for $u_{0}=S\left(\alpha_{0}\right)$.

b. The mapping $S$ satisfies

$$
\left\|S(\alpha)-S\left(\alpha_{0}\right)\right\|_{X} \leq M_{0}\left\|\alpha-\alpha_{0}\right\|_{Z} \quad \forall \alpha \in B\left(\alpha_{0}, \delta\right)
$$

with Lipschitz constant $M_{0}=2 \xi_{0}+1$.

Proof. Define a nonlinear map $A_{\alpha}: X \rightarrow X$ by

$$
A_{\alpha} u:=u-\Gamma_{0} J(\alpha, u) .
$$

We show first that $\exists \varepsilon>0$ such that: (i) $A_{\alpha}$ is a contraction in $B\left(u_{0}, \varepsilon\right)$ and (ii) maps $B\left(u_{0}, \varepsilon\right)$ into itself.

Define $R(\alpha, u):=J(\alpha, u)-J_{u}^{\prime}\left(\alpha_{0}, u_{0}\right)\left(u-u_{0}\right)$. Then from (3.5),

$$
A_{\alpha} u=u_{0}-\Gamma_{0} R(\alpha, u)
$$

and hence by linearity of $\Gamma_{0}$,

$$
A_{\alpha} u-A_{\alpha} u_{1}=-\Gamma_{0}\left(R(\alpha, u)-R\left(\alpha, u_{1}\right)\right) .
$$

For the right-hand side we have by the definition of $R$ and Theorem 2.3 ,

$$
\begin{aligned}
\Gamma_{0}\left(R(\alpha, u)-R\left(\alpha, u_{1}\right)\right) & =\Gamma_{0}\left(J(\alpha, u)-J\left(\alpha, u_{1}\right)-J_{u}^{\prime}\left(\alpha_{0}, u_{0}\right)\left(u-u_{1}\right)\right) \\
& =\int_{0}^{1} \Gamma_{0}\left\{J_{u}^{\prime}\left(\alpha, t u+(1-t) u_{1}\right)-J_{u}^{\prime}\left(\alpha_{0}, u_{0}\right)\right\}\left(u-u_{1}\right) \mathrm{d} t .
\end{aligned}
$$

Then

$$
\begin{aligned}
\frac{\left\|A_{\alpha} u-A_{\alpha} u_{1}\right\|_{X}}{\left\|u-u_{1}\right\|_{X}} & \leq \int_{0}^{1} \eta_{0}\left\{\left\|\alpha-\alpha_{0}\right\|_{Z}+t\left\|u-u_{0}\right\|_{X}+(1-t)\left\|u_{1}-u_{0}\right\|_{X}\right\} \mathrm{d} t \\
& =\eta_{0}\left\{\left\|\alpha-\alpha_{0}\right\|_{Z}+\frac{1}{2}\left\|u-u_{0}\right\|_{X}+\frac{1}{2}\left\|u_{1}-u_{0}\right\|_{X}\right\}=: K .
\end{aligned}
$$

We have $K<1$, i.e., $A_{\alpha}$ is a contraction for any $\alpha \in B\left(\alpha_{0}, \delta\right)$ and $u, u_{1} \in B\left(u_{0}, \varepsilon\right)$, provided that

$$
\eta_{0}(\delta+\varepsilon)<1 \quad \Leftrightarrow \quad \varepsilon \leq \frac{1-\eta_{0} \delta}{\eta_{0}} .
$$

For proving (ii) we need to show

$$
\left\|A_{\alpha} u-u_{0}\right\|_{X} \leq \varepsilon \quad \forall u \in B\left(u_{0}, \varepsilon\right) .
$$


Using (3.5) and (3.6) we have for any $u \in B\left(u_{0}, \varepsilon\right)$,

$$
\begin{aligned}
\left\|A_{\alpha} u-u_{0}\right\|_{X} & \leq\left\|A_{\alpha} u-A_{\alpha} u_{0}\right\|_{X}+\left\|A_{\alpha} u_{0}-u_{0}\right\|_{X} \\
& \leq \eta_{0}\left(\delta+\frac{\varepsilon}{2}\right) \varepsilon+\left\|\Gamma_{0} J\left(\alpha, u_{0}\right)\right\|_{X} .
\end{aligned}
$$

Recalling $J\left(\alpha_{0}, u_{0}\right)=0$ we estimate the last term via triangle inequality

$$
\begin{aligned}
\left\|\Gamma_{0} J\left(\alpha, u_{0}\right)\right\|_{X} & \leq\left\|\Gamma_{0} J_{\alpha}^{\prime}\left(\alpha_{0}, u_{0}\right)\left(\alpha-\alpha_{0}\right)\right\|_{X} \\
& +\left\|\Gamma_{0}\left\{J\left(\alpha, u_{0}\right)-J\left(\alpha_{0}, u_{0}\right)-J_{\alpha}^{\prime}\left(\alpha_{0}, u_{0}\right)\left(\alpha-\alpha_{0}\right)\right\}\right\|_{X} .
\end{aligned}
$$

The first term in the hight-hand side being bounded by $\xi_{0}\left\|\alpha-\alpha_{0}\right\|_{Z}$, the expression in the norm in the last term equals

$$
\int_{0}^{1} \Gamma_{0}\left\{J_{\alpha}^{\prime}\left(s \alpha+(1-s) \alpha_{0}, u_{0}\right)-J_{\alpha}^{\prime}\left(\alpha_{0}, u_{0}\right)\right\}\left(\alpha-\alpha_{0}\right) \mathrm{d} s .
$$

This gives the bound

$$
\begin{aligned}
\left\|\Gamma_{0} J\left(\alpha, u_{0}\right)\right\|_{X} & \leq\left\|\alpha-\alpha_{0}\right\|_{Z}\left(\xi_{0}+\int_{0}^{1} \eta_{0} s\left\|\alpha-\alpha_{0}\right\|_{Z} \mathrm{~d} s\right) \\
& =\left\|\alpha-\alpha_{0}\right\|_{Z}\left(\xi_{0}+\frac{\eta_{0}}{2}\left\|\alpha-\alpha_{0}\right\|_{Z}\right) .
\end{aligned}
$$

Denote $\delta:=\left\|\alpha-\alpha_{0}\right\|_{Z}$, then (3.9) provides

$$
\left\|A_{\alpha} u-u_{0}\right\|_{X} \leq \eta_{0}\left(\delta+\frac{\varepsilon}{2}\right) \varepsilon+\left(\xi_{0}+\frac{\eta_{0}}{2} \delta\right) \delta .
$$

Therefore $A_{\alpha}$ maps $B\left(u_{0}, \varepsilon\right)$ into itself if the right-hand side of (3.10) is bounded by $\varepsilon$, i.e., the radii $\delta, \varepsilon>0$ satisfy

$$
\eta_{0}\left(\delta+\frac{\varepsilon}{2}\right) \varepsilon+\left(\xi_{0}+\frac{\eta_{0}}{2} \delta\right) \delta \leq \varepsilon \quad \Leftrightarrow \quad \tilde{\varepsilon}^{2}-2(1-\tilde{\delta}) \tilde{\varepsilon}+2 \xi_{0} \tilde{\delta}+\tilde{\delta}^{2} \leq 0 .
$$

Here $\tilde{\delta}:=\eta_{0} \delta, \tilde{\varepsilon}:=\eta_{0} \varepsilon$ are the rescaled radii. Resolving (3.11) and using (3.7) we find that $\tilde{\varepsilon} \in \mathcal{U}_{\tilde{\delta}}:=\left[\tilde{\varepsilon}_{-}, \tilde{\varepsilon}_{+}\right] \cap(0,1-\tilde{\delta})$ where

$$
\tilde{\varepsilon}_{ \pm}=1-\tilde{\delta} \pm \sqrt{D} \quad \text { with } \quad D=(1-\tilde{\delta})^{2}-\left(2 \xi_{0} \tilde{\delta}+\tilde{\delta}^{2}\right)=1-2 \tilde{\delta}\left(1+\xi_{0}\right) .
$$

Elementary calculations show that $\mathcal{U}_{\delta} \equiv\left[\tilde{\varepsilon}_{-}, 1-\tilde{\delta}\right)$ is nonempty as soon as $D>0$, since in this case $\tilde{\varepsilon}_{-}>0$ and

$$
\tilde{\delta}<\frac{1}{2\left(1+\xi_{0}\right)}<\frac{1}{2} .
$$

Herewith, we have proven that if $\tilde{\delta}=: \eta_{0} \delta$ satisfies (3.12), i.e., if $\delta<\delta_{0}, \alpha \in U:=$ $B\left(\alpha_{0}, \delta\right)$ and if $\varepsilon_{0}(\delta):=\tilde{\varepsilon}_{-} / \eta_{0}$, then the mapping $A_{\alpha}$ from (3.5) is a contraction on $B\left(u_{0}, \varepsilon_{0}(\delta)\right)$. Furthermore, (3.5) yields that $A_{\alpha}(u)$ is continuous for all $(\alpha, u) \in W$, because $J \in C\left(W, Y^{\prime}\right)$ and $\Gamma_{0}$ is linear. Then the assumptions of [4, Corollary 3.1.4] are satisfied and there exists a unique $S \in C(U, X)$ satisfying $J(\alpha, S(\alpha))=0$ in $U$ and $u_{0}=S\left(\alpha_{0}\right)$.

Next, we prove the estimate (3.4). From the previous considerations, we know in particular that $\alpha \in \partial B\left(\alpha_{0}, \delta\right)$ implies $S(\alpha) \in B\left(u_{0}, \varepsilon_{0}(\delta)\right)$, if $\delta<\delta_{0}$. Then

$$
\left\|S(\alpha)-S\left(\alpha_{0}\right)\right\|_{X} \leq \varepsilon_{0}(\delta)=\frac{1-\tilde{\delta}-\sqrt{1-2 \tilde{\delta}\left(1+\xi_{0}\right)}}{\eta_{0}} \leq \frac{\left(2 \xi_{0}+1\right) \tilde{\delta}}{\eta_{0}} .
$$

Then (3.4) follows, since $\delta=\left\|\alpha-\alpha_{0}\right\|_{Z}=\tilde{\delta} / \eta_{0}$. 
This result does not provide computable quantitative bounds on the size of the neighborhood of $\alpha_{0}$ in which $S$ is differentiable. To obtain such bounds, we require additional assumptions on $J$.

Theorem 3.2. Suppose that the assumptions of Theorem 3.1 are satisfied, that $\delta_{0}$, $\varepsilon_{0}$ are defined as in Theorem 3.1 and that $S: B\left(\alpha_{0}, \delta\right) \rightarrow B\left(u_{0}, \varepsilon_{0}(\delta)\right), \delta<\delta_{0}$ is the solution operator. Assume in addition that:

(1') $J \in C^{1}(W, Z)$ and $J_{\alpha}^{\prime}, J_{u}^{\prime}$ are Lipschitz continuous in $W$.

(2') $J_{u}^{\prime}\left(\alpha_{1}, u_{1}\right)$ with $u_{1}=S\left(\alpha_{1}\right)$ has a uniformly bounded inverse $\Gamma_{1}: Y^{\prime} \rightarrow X$ for any $\alpha_{1}$ in the open ball $\stackrel{\circ}{B}\left(\alpha_{0}, \delta_{0}\right)$.

Define

$$
\xi:=\sup _{\alpha_{1} \in \dot{B}\left(\alpha_{0}, \delta_{0}\right)}\left\|\Gamma_{1} J_{\alpha}^{\prime}\left(\alpha_{1}, S\left(\alpha_{1}\right)\right)\right\|_{Z \rightarrow X}
$$

and $\eta$ being the smallest constant satisfying

$$
\left.\begin{array}{l}
\left\|\Gamma_{1}\left\{J_{\alpha}^{\prime}(\alpha, u)-J_{\alpha}^{\prime}\left(\alpha_{1}, u_{1}\right)\right\}\right\|_{Z \rightarrow X} \\
\left\|\Gamma_{1}\left\{J_{u}^{\prime}(\alpha, u)-J_{u}^{\prime}\left(\alpha_{1}, u_{1}\right)\right\}\right\|_{X \rightarrow X}
\end{array}\right\} \leq \eta\left(\left\|\alpha-\alpha_{1}\right\|_{Z}+\left\|u-u_{1}\right\|_{X}\right)
$$

uniformly for any $(\alpha, u),\left(\alpha_{1}, u_{1}\right) \in W$. Let

$$
\delta_{*}:=\frac{1}{2 \eta(1+\xi)}, \quad \varepsilon_{*}(\delta):=\frac{1-\delta \eta-\sqrt{1-2 \delta \eta(1+\xi)}}{\eta},
$$

and assume that $\delta<\delta_{*} / 2$ is small enough, such that $B\left(\alpha_{0}, \delta\right) \times B\left(u_{0}, \varepsilon_{*}(\delta)\right) \subset W$. Then

(a) $S$ is Lipschitz continuous in $B\left(\alpha_{0}, \delta\right)$, in particular for $M:=2 \xi+1$,

$$
\left\|S(\alpha)-S\left(\alpha_{1}\right)\right\|_{X} \leq M\left\|\alpha-\alpha_{1}\right\|_{Z} \quad \forall \alpha, \alpha_{1} \in B\left(\alpha_{0}, \delta\right) .
$$

(b) $S$ is differentiable in $B\left(\alpha_{0}, \delta\right)$ and

$$
S^{\prime}\left(\alpha_{1}\right)=-\Gamma_{1} J_{\alpha}^{\prime}\left(\alpha_{1}, S\left(\alpha_{1}\right)\right) \quad \forall \alpha_{1} \in B\left(\alpha_{0}, \delta\right) .
$$

(c) $S^{\prime}$ in Lipschitz continuous in $\alpha_{0}$,

$$
\left\|S^{\prime}(\alpha)-S^{\prime}\left(\alpha_{0}\right)\right\|_{Z \rightarrow X} \leq K\left\|\alpha-\alpha_{0}\right\|_{Z} \quad \forall \alpha \in B\left(\alpha_{0}, \delta\right)
$$

with the Lipschitz constant $K=4 \eta_{0}\left(1+\xi_{0}\right)^{2}$.

Proof. (a) If we fix an arbitrary $\alpha_{1} \in B\left(\alpha_{0}, \delta_{0}\right)$, then the assumptions of Theorem 3.1 are satisfied with $\alpha_{1}$ instead of $\alpha_{0}$. We resort to the uniform constants $\xi, \eta$ and obtain the estimate

$$
\left\|S(\alpha)-S\left(\alpha_{1}\right)\right\|_{X} \leq M\left\|\alpha-\alpha_{1}\right\|_{Z} \quad \forall \alpha_{1} \in \stackrel{\circ}{B}\left(\alpha_{0}, \delta_{0}\right), \quad \forall \alpha \in \stackrel{\circ}{B}\left(\alpha_{1}, \delta_{*}\right)
$$

with $M=2 \xi+1$. Suppose $\alpha, \alpha_{1} \in B\left(\alpha_{0}, \delta\right)$ with $\delta<\delta_{*} / 2$. Then $\alpha_{1} \in \stackrel{\circ}{B}\left(\alpha_{0}, \delta_{0}\right)$ and $\alpha \in \stackrel{\circ}{B}\left(\alpha_{1}, \delta_{*}\right)$, since

$\left\|\alpha_{1}-\alpha_{0}\right\|_{Z} \leq \delta_{*} / 2<\delta_{0} \quad$ and $\quad\left\|\alpha-\alpha_{1}\right\|_{Z} \leq\left\|\alpha-\alpha_{0}\right\|_{Z}+\left\|\alpha_{1}-\alpha_{0}\right\|_{Z} \leq 2 \delta<\delta_{*}$ which together with (3.20) yields 3.17).

(b) We prove differentiability of $S$ in $B\left(\alpha_{0}, \delta\right)$. To this end we fix an arbitrary element $\alpha_{1} \in B\left(\alpha_{0}, \delta\right)$ and define

$$
u_{1}:=S\left(\alpha_{1}\right), \quad \Gamma_{1}:=\left[J_{u}^{\prime}\left(\alpha_{1}, u_{1}\right)\right]^{-1}, \quad \Lambda_{1}:=-\Gamma_{1} J_{\alpha}^{\prime}\left(\alpha_{1}, u_{1}\right) .
$$


Recall $J\left(\alpha_{1}, u_{1}\right)=0=J(\alpha, u)$. Then we have for $u:=S(\alpha)$,

$$
\begin{array}{r}
\left\|S(\alpha)-S\left(\alpha_{1}\right)-\Lambda_{1}\left(\alpha-\alpha_{1}\right)\right\|_{X}=\left\|\Gamma_{1}\left\{J_{\alpha}^{\prime}\left(\alpha_{1}, u_{1}\right)\left(\alpha-\alpha_{1}\right)-J_{u}^{\prime}\left(\alpha_{1}, u_{1}\right)\left(u-u_{1}\right)\right\}\right\|_{X} \\
\leq \int_{0}^{1}\left\|\Gamma_{1}\left(J_{\alpha}^{\prime}\left(s \alpha+(1-s) \alpha_{1}, u\right)-J_{\alpha}^{\prime}\left(\alpha_{1}, u_{1}\right)\right)\right\|_{Z \rightarrow X}\left\|\alpha-\alpha_{1}\right\|_{Z} \mathrm{~d} s \\
+\int_{0}^{1}\left\|\Gamma_{1}\left(J_{u}^{\prime}\left(\alpha_{1}, t u+(1-t) u_{1}\right)-J_{u}^{\prime}\left(\alpha_{1}, u_{1}\right)\right)\right\|_{X \rightarrow X}\left\|u-u_{1}\right\|_{X} \mathrm{~d} t \\
\leq \eta\left\{\left(\frac{1}{2}\left\|\alpha-\alpha_{1}\right\|_{Z}+\left\|u-u_{1}\right\|_{X}\right)\left\|\alpha-\alpha_{1}\right\|_{Z}+\frac{1}{2}\left\|u-u_{1}\right\|_{X}^{2}\right\} \\
\leq \frac{\eta}{2}(1+M)^{2}\left\|\alpha-\alpha_{1}\right\|_{Z}^{2}
\end{array}
$$

by Lipschitz continuity of $S$ in $B\left(\alpha_{0}, \delta\right)$; cf. (3.17). The right-hand side is $\mathcal{O}(\| \alpha-$ $\left.\alpha_{1} \|_{Z}^{2}\right)$, thus by Definition 2.1, $S$ is differentiable in any $\alpha_{1} \in B\left(\alpha_{0}, \delta\right)$ and $S^{\prime}\left(\alpha_{1}\right) \equiv$ $\Lambda_{1}$.

(c) Finally, we prove that $S^{\prime}$ is Lipschitz continuous in $u_{0}$. Relation (3.18) is equivalent to

$$
J_{u}^{\prime}(\alpha, S(\alpha)) S^{\prime}(\alpha)+J_{\alpha}^{\prime}(\alpha, S(\alpha))=0, \quad \forall \alpha \in B\left(\alpha_{0}, \delta\right) .
$$

Then for $u=S(\alpha), u_{0}=S\left(\alpha_{0}\right)$ we have the identity

$$
\begin{aligned}
&\left(J_{u}^{\prime}(\alpha, u)-J_{u}^{\prime}\left(\alpha_{0}, u_{0}\right)\right) S^{\prime}(\alpha)+J_{u}^{\prime}\left(\alpha_{0}, u_{0}\right)\left(S^{\prime}(\alpha)-S^{\prime}\left(\alpha_{0}\right)\right) \\
&+J_{\alpha}^{\prime}(\alpha, u)-J_{\alpha}^{\prime}\left(\alpha_{0}, u_{0}\right)=0 .
\end{aligned}
$$

This yields with $\Gamma_{0}=\left[J_{u}^{\prime}\left(\alpha_{0}, u_{0}\right)\right]^{-1}$,

$$
\begin{aligned}
\left\|S^{\prime}(\alpha)-S^{\prime}\left(\alpha_{0}\right)\right\|_{Z \rightarrow X} \leq & \left\|\Gamma_{0}\left(J_{u}^{\prime}(\alpha, u)-J_{u}^{\prime}\left(\alpha_{0}, u_{0}\right)\right)\right\|_{X \rightarrow X}\left\|S^{\prime}(\alpha)\right\|_{Z \rightarrow X} \\
& +\left\|\Gamma_{0}\left(J_{\alpha}^{\prime}(\alpha, u)-J_{\alpha}^{\prime}\left(\alpha_{0}, u_{0}\right)\right)\right\|_{Z \rightarrow X} \\
\leq & \eta_{0}\left(\left\|\alpha-\alpha_{0}\right\|_{Z}+\left\|S(\alpha)-S\left(\alpha_{0}\right)\right\|_{X}\right)\left(1+\left\|S^{\prime}(\alpha)\right\|_{Z \rightarrow X}\right) \\
\leq & \Theta\left\|\alpha-\alpha_{0}\right\|_{Z}\left(1+\left\|S^{\prime}\left(\alpha_{0}\right)\right\|_{Z \rightarrow X}+\left\|S^{\prime}(\alpha)-S^{\prime}\left(\alpha_{0}\right)\right\|_{Z \rightarrow X}\right)
\end{aligned}
$$

with $\Theta=\eta_{0}\left(M_{0}+1\right)=2 \eta_{0}\left(1+\xi_{0}\right)=1 / \delta_{0}$. Assume $\alpha \in B\left(\alpha_{0}, \delta\right), \delta<\delta_{*} / 2$. Then $\Theta\left\|\alpha-\alpha_{0}\right\|_{Z}<\delta_{*} /\left(2 \delta_{0}\right) \leq 1 / 2$. This implies

$$
\left\|S^{\prime}(\alpha)-S^{\prime}\left(\alpha_{0}\right)\right\|_{Z \rightarrow X} \leq 2 \Theta\left(1+\xi_{0}\right)\left\|\alpha-\alpha_{0}\right\|_{Z} .
$$

The proof is complete.

\section{RANDOM FIELDS UNDER NONLINEAR MAPS}

In this section we study properties of random fields and their characteristics under nonlinear maps and apply it to solution operators of the implicit nonlinear equation (1.1). We establish measurability (Lemma 4.1) and summability (Lemma 4.2) properties of random fields under nonlinear maps. In combination with Theorem 2.4. this yields existence of the moments of the solution in a sufficiently small neighborhood $U$ about the nominal parameter value $\alpha_{0}$; cf. Theorem 4.6 and the asymptotic approximation result (4.16). Theorems 3.1 and 3.2 from the previous section allow us to control the size of $U$. Based on this, we obtain nonasymptotic approximation bounds in Theorem 4.8 which allow for $\alpha(\omega)$ belonging to $U$ almost 
surely (a.s.) where $U$ is not necessarily small. The key property here is Lipschitz continuity of the solution operator; cf. Lemma 4.7

We begin with basic definitions; see e.g. [10, 19, 18. Let $(\Omega, \mathcal{A}, \mathbb{P})$ be a probability space where $\Omega$ is a set of elementary events, $\mathcal{A} \subset 2^{\Omega}$ is a set of all possible events being a $\sigma$-algebra over $\Omega$, and $\mathbb{P}$ is a probability measure. If $(X, \mathcal{F})$ and $(Y, \mathcal{G})$ are measurable spaces, a map $f: X \rightarrow Y$ is called $\mathcal{F}$ - $\mathcal{G}$-measurable, if $f^{-1}(\mathcal{G}) \subseteq \mathcal{F}$. For a complete metric space $E$, we shall denote by $\mathcal{B}(E)$ the Borel $\sigma$-algebra of $E$ generated by all open subsets of $E$. A mapping $\alpha: \Omega \rightarrow E$ is called a random variable if it is $\mathcal{F}-\mathcal{B}(E)$-measurable. In this paper we are interested in random variables with values in Banach spaces. From now we assume that $X, Y$ and $Z$ are Banach spaces.

Under the above notations we introduce the set of all $\mathcal{A}$-measurable functions

$$
L^{0}(\Omega ; Z):=\{\alpha: \Omega \rightarrow Z: \alpha \text { is } \mathcal{A}-\mathcal{B}(Z) \text {-measurable }\},
$$

that is, the set of all random variables $\Omega \rightarrow Z$. Suppose $\alpha \in L^{0}(\Omega ; Z)$ and $S: Z \rightarrow$ $X$ is a general nonlinear map (in what follows, $S$ shall be interpreted as the solution operator from Section 2). Sufficient conditions under which the composition $S \circ \alpha$ is a random variable, are given by the following classical result.

Lemma 4.1. Suppose $X, Z$ are Banach spaces, $\alpha \in L^{0}(\Omega ; Z)$ and $S: Z \rightarrow X$. The mapping $u:=S \circ \alpha: \Omega \rightarrow X$ is a random variable if $S$ is Borel measurable, that is, $\mathcal{B}(Z)-\mathcal{B}(X)$-measurable. A sufficient condition is $S \in C(\alpha(\Omega), X)$.

Proof. We establish sufficiency. As $S$ is continuous, $S^{-1}(V)$ is open in $\alpha(\Omega)$ for any $V \in \mathcal{B}(X)$, i.e., $\exists U \in \mathcal{B}(Z): S^{-1}(V)=\alpha(\Omega) \cap U$. Then

$$
(S \circ \alpha)^{-1}(V)=\alpha^{-1}\left(S^{-1}(V)\right)=\alpha^{-1}(\alpha(\Omega) \cap U)=\alpha^{-1}(U) \in \mathcal{A},
$$

since $\alpha \in L^{0}(\Omega ; Z)$ by assumption.

A function $\alpha: \Omega \rightarrow Z$ is called $\mathbb{P}$-almost surely separable valued if there exists a $\mathbb{P}$-null set $N$ such that the image of its complement $\alpha(\Omega \backslash N)$ is separable. In order to introduce a Bochner-Lebesgue integral we resort to a subspace of $\mathbb{P}$-measurable random variables, which can be equivalently defined by

$$
L^{0}(\Omega, \mathbb{P} ; Z):=\left\{\alpha \in L^{0}(\Omega ; Z): \alpha \text { is } \mathbb{P} \text {-almost surely separable valued }\right\} .
$$

The space $L^{0}(\Omega, \mathbb{P} ; Z)$ is also called a Bochner space of strongly measurable functions. For a Banach space $Z$ we define

$$
\|\alpha\|_{L^{k}(\Omega, \mathbb{P} ; Z)}:=\left\{\begin{array}{cc}
\left(\int_{\Omega}\|\alpha(\omega)\|_{Z}^{k} \mathrm{dP}(\omega)\right)^{1 / k} & 1 \leq k<\infty, \\
\underset{\omega \in \Omega}{\operatorname{ess} \sup _{\omega \in \Omega}\|\alpha(\omega)\|_{Z}} & k=\infty
\end{array}\right.
$$

and $\mathbb{E}[\cdot]:=\int_{\Omega}(\cdot) \mathrm{d} \mathbb{P}(\omega)$ with the integral denoting the Bochner-Lebesgue integral. Then

$$
L^{k}(\Omega, \mathbb{P} ; Z):=\left\{\alpha \in L^{0}(\Omega, \mathbb{P} ; Z):\|\alpha\|_{L^{k}(\Omega, \mathbb{P} ; Z)}<\infty\right\} / \mathcal{N}
$$

where $\mathcal{N}=\left\{\alpha \in L^{0}(\Omega, \mathbb{P} ; Z): \alpha=0 \mathbb{P}\right.$-a.e. $\}$. The Bochner-Lebesgue space $L^{k}(\Omega, \mathbb{P} ; Z)$ is a Banach space with the norm (4.3).

Throughout our analysis of random perturbations, we assume w.l.o.g. that the nominal parameter value $\alpha_{0} \in U \subset Z$ in our linearization of the solution map $S(\alpha)$ is the mean field of $\alpha(\omega)$ or, equivalently, that the mean field of the parameter fluctuations $r(\omega)=\alpha(\omega)-\alpha_{0}$ vanishes. 
Lemma 4.2. Suppose $X, Z$ are Banach spaces, $\alpha \in L^{k}(\Omega, \mathbb{P} ; Z)$ and that $S: Z \rightarrow$ $X$ is Lipschitz continuous at $\alpha_{0} \in Z$ : there exists an open neighborhood $U \subseteq Z$ of $\alpha_{0}$ and a constant $M>0$, such that

$$
\left\|S(\tilde{\alpha})-S\left(\alpha_{0}\right)\right\|_{X} \leq M\left\|\tilde{\alpha}-\alpha_{0}\right\|_{Z} \quad \forall \tilde{\alpha} \in U .
$$

Assume in addition that

$$
\mathbb{P}\{\alpha \in U\}=1
$$

Then $u=S(\alpha) \in L^{k}(\Omega, \mathbb{P} ; X)$.

Proof. Suppose $1 \leq k<\infty$. Then

$$
\left\|S(\alpha)-S\left(\alpha_{0}\right)\right\|_{L^{k}(\Omega, \mathbb{P} ; X)} \leq M\left\|\alpha-\alpha_{0}\right\|_{L^{k}(\Omega, \mathbb{P} ; Z)}
$$

for any $\alpha(\omega)$ belonging to $U \mathbb{P}$-almost surely, since

$$
\int_{\Omega}\left\|S(\alpha)-S\left(\alpha_{0}\right)\right\|_{X}^{k} \mathrm{~d} \mathbb{P}(\omega) \leq M^{k} \int_{\Omega}\left\|\alpha-\alpha_{0}\right\|_{Z}^{k} \mathrm{~d} \mathbb{P}(\omega) .
$$

From (4.5) we infer that (4.7) holds also for $k=\infty$.

In what follows we shall introduce and study properties of $k$-th statistical moments. For that it is convenient to introduce the following abbreviations for tensor products.

Definition 4.3. For any two Banach spaces $X, Y$ we denote by $X \otimes Y$ its tensor product being a Banach space endowed with a reasonable cross-norm satisfying

$$
\|u \otimes v\|_{X \otimes Y}=\|u\|_{X}\|v\|_{Y}, \quad \forall u \in X, v \in Y .
$$

The $k$-fold tensor product $X \otimes \cdots \otimes X$ is defined by induction and is denoted by $X^{(k)}$. Analogously, for an element $u \in X$ we define its $k$-fold product $u^{(k)}:=$ $u \otimes \cdots \otimes u \in X^{(k)}$; for a bounded linear operator $A \in \mathcal{L}(X, Y)$ we define its $k$-fold tensor product $A^{(k)}=A \otimes \cdots \otimes A \in \mathcal{L}\left(X^{(k)}, Y^{(k)}\right)$.

Definition 4.4. Let $k \geq 1$ be an integer. Then for $\alpha \in L^{k}(\Omega, \mathbb{P} ; Z)$ its $k$-th statistical moment is defined by

$$
\mathcal{M}^{k} \alpha=\mathbb{E}\left[\alpha^{(k)}\right]=\int_{\Omega} \alpha^{(k)} \mathrm{d} \mathbb{P}(\omega) \in Z^{(k)} .
$$

Note that $\mathcal{M}^{k}$ is well defined as a mapping $L^{k}(\Omega, \mathbb{P} ; Z) \rightarrow Z^{(k)}$, since

$$
\left\|\mathcal{M}^{k} \alpha\right\|_{Z^{(k)}} \leq\left\|\alpha^{(k)}\right\|_{L^{1}\left(\Omega, \mathbb{P} ; Z^{(k)}\right)}=\|\alpha\|_{L^{k}(\Omega, \mathbb{P} ; Z)}^{k} .
$$

Corollary 4.5. Suppose $X, Y, Z$ are Banach spaces and $W$ is a neighborhood of $\left(\alpha_{0}, u_{0}\right) \in Z \times X$. Let $\alpha \in L^{0}(\Omega ; Z)$ be a random variable and consider a mapping $J$ as defined in (1.2). Suppose $J\left(\alpha_{0}, u_{0}\right)=0$ and:

(1) $J \in C\left(W, Y^{\prime}\right)$ and its differential $J_{u}^{\prime}(\alpha, u) \in \mathcal{L}\left(X, Y^{\prime}\right)$ exist in $W$ and are continuous in $\left(\alpha_{0}, u_{0}\right)$.

(2) The "sensitivity operator" $G_{0}:=J_{u}^{\prime}\left(\alpha_{0}, u_{0}\right)$ admits a bounded inverse $\Gamma_{0} \in$ $\mathcal{L}\left(Y^{\prime}, X\right)$.

Then there is a neighborhood $U$ of $\alpha_{0}$ and a unique random variable $u \in L^{0}(\Omega ; X)$ such that $u=S(\alpha)$ satisfies (1.1) if $\mathbb{P}\{\alpha(\omega) \in U\}=1$, and $S\left(\alpha_{0}\right)=u_{0}$.

Suppose (1) is replaced with the stronger assumption:

(1') $J \in C\left(W, Y^{\prime}\right), J_{\alpha}^{\prime} \in \mathcal{L}\left(Z, Y^{\prime}\right), J_{u}^{\prime} \in \mathcal{L}\left(X, Y^{\prime}\right)$ exist in $W$ and are Lipschitz continuous in $\left(\alpha_{0}, u_{0}\right)$. 
Then

(a) $U$ can be chosen as $U=B\left(\alpha_{0}, \delta\right), \delta<\delta_{0}$ with $\delta_{0}$ from Theorem 3.1

(b) If $\mathbb{P}\left\{\left\|\alpha(\omega)-\alpha_{0}\right\|_{Z} \leq \delta\right\}=1$, then $\alpha \in L^{k}(\Omega, \mathbb{P} ; Z)$ and $u \in L^{k}(\Omega, \mathbb{P} ; X)$ for any $1 \leq k \leq \infty$.

Proof. Existence of a unique solution operator $S \in C(U, X)$ follows from Theorem 2.4. Lemma 4.1 yields that $u=S(\alpha)$ is $\mathcal{A}-\mathcal{B}(X)$-measurable. Theorem 3.1 yields (a). Assertion (b) follows from

$$
\left\|\alpha-\alpha_{0}\right\|_{L^{k}(\Omega, \mathbb{P} ; Z)} \leq \delta_{0}, \quad\left\|u-u_{0}\right\|_{L^{k}(\Omega, \mathbb{P} ; X)} \leq M \delta_{0}
$$

guaranteed by (3.4) and Lemma 4.2

We see from Corollary 4.5 that $u \in L^{k}(\Omega, \mathbb{P} ; X)$ for any $k$, if $J_{\alpha}^{\prime}, J_{u}^{\prime}$ are Lipschitz continuous at $\left(\alpha_{0}, u_{0}\right)$ and the random parameter $\alpha(\omega)$ belongs $\mathbb{P}$-a.s. to a sufficiently small (bounded) neighborhood of $\alpha_{0} \in Z$. This assumption is however of limited use in practice. In particular, $\alpha$ cannot be modeled as a Gaussian random field taking arbitrary large values with small but positive probability. This difficulty cannot be overcome in general due to the nonlinear nature of $J$, e.g., the implicit equation (1.1) might be not uniquely solvable for certain values of $\alpha$ far from $\alpha_{0}$. Fortunately, for some classes of $J$ unbounded values of $\alpha$ can be allowed, as we show in Lemma 4.7 below.

Considering models with such unbounded random parameters is possible if $J$ is linear and continuous [24, 26, 7]. In this case the solution operator $S$ is linear and

$$
\mathcal{M}^{k} u=\mathbb{E}[S(\alpha) \otimes \cdots \otimes S(\alpha)]=(S \otimes \cdots \otimes S) \mathcal{M}^{k} \alpha .
$$

In the linear case we obtain from (1.5)

$$
J_{u}^{\prime} u=-J_{\alpha}^{\prime} \alpha \Longleftrightarrow u=S(\alpha)=S^{\prime} \alpha=-\left[J_{u}^{\prime}\right]^{-1} J_{\alpha}^{\prime} \alpha,
$$

where $S^{\prime}, J_{u}^{\prime}, J_{\alpha}^{\prime}$ do not depend on $(\alpha, u)$. If $J_{u}^{\prime}$ is invertible, then $\alpha \in L^{k}(\Omega, \mathbb{P} ; Z)$ implies $u \in L^{k}(\Omega, \mathbb{P} ; X)$ and hence the $k$-point correlation function to first order, $\mathcal{M}^{k} u$, is well defined in $X^{(k)}$ by (4.10). In this case sparse tensor schemes can be constructed for approximate numerical evaluation of $S=-\left[J_{u}^{\prime}\right]^{-1} J_{\alpha}^{\prime}$; see $[24,26,7]$.

In what follows we shall generalize this idea to a class of nonlinear problems via local implicit problems of the type (1.1). The starting point is to rewrite (4.11) in view of (4.12) in the form

$$
\mathcal{M}^{k} u=\mathcal{M}^{k} S^{\prime} \alpha=\left(S^{\prime} \otimes \cdots \otimes S^{\prime}\right) \mathcal{M}^{k} \alpha .
$$

Here both $S$ and $S^{\prime}$ are linear operators, and $S^{\prime}$ does not depend on the evaluation point $\alpha_{0}$; see (2.1). If $S$ is nonlinear, then $S^{\prime}$ depends on the evaluation point $\alpha_{0}$ and (4.13) is no longer true. However, $S^{\prime}\left(\alpha_{0}\right) \in \mathcal{L}(Z, X)$ is still a bounded, linear operator and the nonlinear version of (4.13) reads

$$
\mathcal{M}^{k}\left[u-u_{0}\right] \approx \mathcal{M}^{k}\left[S^{\prime}\left(\alpha_{0}\right)\left(\alpha-\alpha_{0}\right)\right]=\left(S^{\prime}\left(\alpha_{0}\right) \otimes \cdots \otimes S^{\prime}\left(\alpha_{0}\right)\right) \mathcal{M}^{k}\left[\alpha-\alpha_{0}\right] .
$$

In other words, $\mathcal{M}^{k}\left[S^{\prime}\left(\alpha_{0}\right)\left(\alpha-\alpha_{0}\right)\right]$ can be computed pursuing the same strategy as in the linear case and requires only the knowledge of $\mathcal{M}^{k}\left[\alpha-\alpha_{0}\right]$ and of a numerical procedure of approximate evaluation of the first variation at the nominal pair $\left(\alpha_{0}, u_{0}\right)$,

$$
S^{\prime}\left(\alpha_{0}\right)=-\left[J_{u}^{\prime}\left(\alpha_{0}, u_{0}\right)\right]^{-1} J_{\alpha}^{\prime}\left(\alpha_{0}, u_{0}\right)=-\Gamma_{0} J_{\alpha}^{\prime}\left(\alpha_{0}, u_{0}\right) .
$$


Since the exact inverse $\Gamma_{0}$ is usually not available, we interpret (4.14) as the first order tensorized operator equation for the $k$-th moment $\mathcal{M}^{k}\left[u-u_{0}\right]$, i.e., we consider the tensorized equation

$$
\text { find } Z^{(k)} \in X^{(k)}: \quad G_{0}^{(k)} Z^{(k)}=(-1)^{k}\left[J_{\alpha}^{\prime}\left(\alpha_{0}, u_{0}\right)\right]^{(k)} \mathcal{M}^{k}\left[\alpha-\alpha_{0}\right]
$$

where $G_{0}=J_{u}^{\prime}\left(\alpha_{0}, u_{0}\right)$. The (linear) equation (4.15) for the $k$-th moment of the random solution can be solved approximately by Galerkin projection of (4.15) onto tensorized finite element spaces, thus giving rise to the terminology "first order $k$ th-moment FE analysis". Since (4.15) was derived by (first order) linearization and tensorization of the tangent, or "sensitivity" operator, this strategy will incur two types of errors: first, a linearization error and second, a Galerkin approximation error. On the other hand, it allows to compute information on statistical moments of the random response $u(\omega)$ deterministically, i.e., without sampling the operator equation.

Based on (4.14), the solution $Z^{(k)}=\mathcal{M}^{k}\left[S^{\prime}\left(\alpha_{0}\right)\left(\alpha-\alpha_{0}\right)\right]$ of (4.15) should be a good approximation of $\mathcal{M}^{k}\left[u-u_{0}\right]$. By the definition of the Fréchet derivative (2.1) we infer that the approximation must be good at least if $\alpha$ belongs to a sufficiently small neighborhood of $\alpha_{0}$ as the following asymptotic result shows.

Theorem 4.6. Suppose that the functional $J$ and its derivatives satisfy the assumptions of Theorem 3.2 and $\mathbb{P}\left\{\left\|\alpha(\omega)-\alpha_{0}\right\|_{Z} \leq \delta\right\}=1$. Then

$$
\left\|\mathcal{M}^{k}\left[u-u_{0}\right]-\mathcal{M}^{k}\left[S^{\prime}\left(\alpha_{0}\right)\left(\alpha-\alpha_{0}\right)\right]\right\|_{X^{(k)}}=o\left(\delta^{k}\right), \quad \delta \rightarrow 0 .
$$

Proof. Due to Theorem 3.2, the solution operator $S$ exists and is differentiable in $B\left(\alpha_{0}, \delta\right)$ for $\delta<\delta_{*} / 2$. According to (2.1) we have for $u=S(\alpha), u_{0}=S\left(\alpha_{0}\right)$ and $r=\alpha-\alpha_{0}$,

$$
u=u_{0}+S^{\prime}\left(\alpha_{0}\right) r+R_{0}(r), \quad\left\|R_{0}(r)\right\|_{X}=o\left(\|r\|_{Z}\right) .
$$

Due to (4.9),

$$
\mathcal{M}^{k}\left[u-u_{0}\right]-\mathcal{M}^{k}\left[S^{\prime}\left(\alpha_{0}\right) r\right]=\mathbb{E}\left[\left(S^{\prime}\left(\alpha_{0}\right) r+R_{0}(r)\right)^{(k)}\right]-\mathbb{E}\left[\left(S^{\prime}\left(\alpha_{0}\right) r\right)^{(k)}\right] .
$$

Hence, by property of the cross-norm (4.8) and the binomial theorem

$$
\begin{array}{r}
\left\|\mathcal{M}^{k}\left[u-u_{0}\right]-\mathcal{M}^{k}\left[S^{\prime}\left(\alpha_{0}\right) r\right]\right\|_{X^{(k)}} \leq \mathbb{E}\left[\sum_{i=1}^{k}\left(\begin{array}{c}
k \\
i
\end{array}\right)\left\|S^{\prime}\left(\alpha_{0}\right) r\right\|_{X}^{k-i}\left\|R_{0}(r)\right\|_{Z}^{i}\right] \\
\leq \mathbb{E}\left[\sum_{i=1}^{k}\left(\begin{array}{c}
k \\
i
\end{array}\right)\left\|S^{\prime}\left(\alpha_{0}\right)\right\|_{Z \rightarrow X}^{k-i}\|r\|_{Z}^{k-i}\left\|R_{0}(r)\right\|_{Z}^{i}\right] \\
\leq \mathbb{E}\left[\|r\|_{Z}^{k}\right] \sum_{i=1}^{k}\left(\begin{array}{c}
k \\
i
\end{array}\right) \xi_{0}^{k-i} \sup _{\|\tilde{r}\|_{Z} \leq \delta}\left(\frac{\left\|R_{0}(\tilde{r})\right\|_{X}}{\|\tilde{r}\|_{Z}}\right)^{i} .
\end{array}
$$

If $\mathbb{P}\left\{\|r(\omega)\|_{Z} \leq \delta\right\}=1$ we have $\mathbb{E}\left[\|r\|_{Z}^{k}\right] \leq \delta^{k}$ and

$$
\frac{\left\|\mathcal{M}^{k}\left[u-u_{0}\right]-\mathcal{M}^{k}\left[S^{\prime}\left(\alpha_{0}\right) r\right]\right\|_{X^{(k)}}}{\delta^{k}} \rightarrow 0, \quad \delta \rightarrow 0 .
$$

The proof is complete.

The approximation (4.14) could be accurate even for large, possibly unbounded values for $\alpha$, provided such values occur with a small probability. For example, such that the perturbation $\alpha-\alpha_{0}$ is $L^{m}$-summable for $m \geq k$ sufficiently high. The essential requirement here is local Lipschitz continuity of the Fréchet derivative of 
the solution operator on the possibly unbounded set containing $\alpha_{0}$ in the sense of (4.17). We give this result first for a nonlinear sufficiently smooth mapping $F$ in the following lemma.

Lemma 4.7. Suppose $X, Z$ are Banach spaces, $\emptyset \neq U \subseteq Z$ is open and $\alpha_{0} \in U$. Let $F \in C^{1}(U, X)$ be a nonlinear map for which $F^{\prime}$ is Lipschitz continuous at $\alpha_{0}$ in the following sense:

$$
\exists K>0: \quad\left\|F^{\prime}(\tilde{\alpha})-F^{\prime}\left(\alpha_{0}\right)\right\|_{Z \rightarrow X} \leq K\left\|\tilde{\alpha}-\alpha_{0}\right\|_{Z} \quad \forall \tilde{\alpha} \in U .
$$

Assume that $B:=\left\|F^{\prime}\left(\alpha_{0}\right)\right\|_{Z \rightarrow X}<\infty$ and let $\alpha \in L^{0}(\Omega ; Z)$ be a random field satisfying $\mathbb{P}\{\alpha(\omega) \in U\}=1$.

(1) If $\alpha \in L^{2 k}(\Omega, \mathbb{P} ; Z)$ for some $k \in \mathbb{N}$, then

$$
\begin{aligned}
& \left\|\mathcal{M}^{k}\left[F(\alpha)-F\left(\alpha_{0}\right)\right]-\mathcal{M}^{k}\left[F^{\prime}\left(\alpha_{0}\right)\left(\alpha-\alpha_{0}\right)\right]\right\|_{X^{(k)}} \\
& \quad \leq\left((B+K / 2)^{k}-B^{k}\right) \max _{m \in\{k+1, \ldots, 2 k\}}\left\|\alpha-\alpha_{0}\right\|_{L^{m}(\Omega, \mathbb{P} ; Z)}^{m} .
\end{aligned}
$$

(2) If $\alpha \in L^{k+1}(\Omega, \mathbb{P} ; Z)$, then

$$
\begin{array}{r}
\left\|\mathcal{M}^{k}\left[F(\alpha)-F\left(\alpha_{0}\right)\right]-\mathcal{M}^{k}\left[F^{\prime}\left(\alpha_{0}\right)\left(\alpha-\alpha_{0}\right)\right]\right\|_{X^{(k)}} \\
\leq \frac{k}{2} K B_{*}^{k-1}\left\|\alpha-\alpha_{0}\right\|_{L^{k+1}(\Omega, \mathbb{P} ; Z)}^{k+1},
\end{array}
$$

where $B_{*}:=\sup _{\tilde{\alpha} \in U}\left\|F^{\prime}(\tilde{\alpha})\right\|_{Z \rightarrow X}<\infty$.

Proof. (1) Let $r:=\alpha-\alpha_{0}$ be a random increment such that $r(\omega) \in U-\alpha_{0} \mathbb{P}-$ almost surely. In particular, large realizations $r(\omega)$ are allowed, if $U \subseteq Z$ is large. By Theorem 2.3 we have in this case

$$
F(\alpha)-F\left(\alpha_{0}\right)=\int_{0}^{1} F^{\prime}\left(\alpha_{0}+r \theta\right) r \mathrm{~d} \theta
$$

This yields

$$
\begin{aligned}
\mathcal{M}^{k}\left[F(\alpha)-F\left(\alpha_{0}\right)\right] & =\mathbb{E}\left[\left(\int_{0}^{1} F^{\prime}\left(\alpha_{0}+r(\omega) \theta\right) r(\omega) \mathrm{d} \theta\right)^{(k)}\right] \\
& =\mathbb{E}\left[\int_{[0,1]^{k}} \prod_{i=1}^{k} F^{\prime}\left(\alpha_{0}+r(\omega) \theta_{i}\right) r(\omega) \mathrm{d} \theta_{1} \ldots \mathrm{d} \theta_{k}\right] .
\end{aligned}
$$

Thus

$$
\begin{aligned}
& \mathcal{M}^{k}\left[F(\alpha)-F\left(\alpha_{0}\right)\right]-\mathcal{M}^{k}\left[F^{\prime}\left(\alpha_{0}\right) r\right] \\
& =\mathbb{E}\left[\int_{[0,1]^{k}}\left\{\prod_{i=1}^{k} F^{\prime}\left(\alpha_{0}+r(\omega) \theta_{i}\right) r(\omega)-\prod_{i=1}^{k} F^{\prime}\left(\alpha_{0}\right) r(\omega)\right\} \mathrm{d} \theta_{1} \ldots \mathrm{d} \theta_{k}\right] .
\end{aligned}
$$

For any fixed $\omega \in \Omega$ and $\theta_{1}, \ldots, \theta_{k} \in[0,1]$ the term in the inner brackets is equal to

$$
\sum_{j=1}^{k}\left(\prod_{i=1}^{j-1} F^{\prime}\left(\alpha_{0}+r \theta_{i}\right) r\right)\left(F^{\prime}\left(\alpha_{0}+r \theta_{j}\right) r-F^{\prime}\left(\alpha_{0}\right) r\right)\left(\prod_{i=j+1}^{k} F^{\prime}\left(\alpha_{0}\right) r\right)=: \Delta(\omega) .
$$


Recalling the definition of $K, B$ we find $\left\|F^{\prime}(\tilde{\alpha})\right\|_{Z \rightarrow X} \leq B+K\left\|\tilde{\alpha}-\alpha_{0}\right\|_{Z}$ for any $\tilde{\alpha} \in U$. Thus we obtain by triangle inequality and by the definition (4.8) of the cross-norm

$$
\|\Delta(\omega)\|_{X^{(k)}} \leq\|r(\omega)\|_{X}^{k} \sum_{j=1}^{k}\left(\prod_{i=1}^{j-1}\left(B+K \theta_{i}\|r(\omega)\|_{Z}\right)\right)\left(K \theta_{j}\|r(\omega)\|_{Z}\right) B^{k-j}
$$

Inserting into (4.20) and integrating over $\theta_{1}, \ldots, \theta_{k}$ we conclude

$$
\begin{aligned}
& \| \mathcal{M}^{k}\left[F(\alpha)-F\left(\alpha_{0}\right)\right]-\mathcal{M}^{k}\left[F^{\prime}\left(\alpha_{0}\right) r\right] \|_{X^{(k)}} \leq \mathbb{E}\left[\int_{[0,1]^{k}}\|\Delta(\omega)\|_{X^{(k)}} \mathrm{d} \theta_{1} \ldots \mathrm{d} \theta_{k}\right] \\
& \leq \mathbb{E}\left[\|r(\omega)\|_{Z}^{k} \sum_{j=1}^{k}\left(B+\frac{K}{2}\|r(\omega)\|_{Z}\right)^{j-1} \frac{K}{2}\|r(\omega)\|_{Z} B^{k-j}\right] .
\end{aligned}
$$

By the binomial theorem the right-hand side is equal to

$$
\begin{aligned}
& =\mathbb{E}\left[\frac{K}{2}\|r(\omega)\|_{Z}^{k+1} \sum_{j=1}^{k} B^{k-j} \sum_{i=0}^{j-1}\left(\begin{array}{c}
j-1 \\
i
\end{array}\right)\left(\frac{K}{2}\|r(\omega)\|_{Z}\right)^{i} B^{j-i-1}\right] \\
& =\sum_{j=1}^{k} \sum_{i=0}^{j-1}\left(\begin{array}{c}
j-1 \\
i
\end{array}\right)\left(\frac{K}{2}\right)^{i+1} B^{k-i-1} \mathbb{E}\left[\|r(\omega)\|_{Z}^{k+i+1}\right] .
\end{aligned}
$$

In this expression only the binomial coefficient depends on $j$. Interchanging the summation order we find for any numbers $g_{i} \in \mathbb{R}$,

$$
\sum_{j=1}^{k} \sum_{i=0}^{j-1}\left(\begin{array}{c}
j-1 \\
i
\end{array}\right) g_{i}=\sum_{j=0}^{k-1} \sum_{i=0}^{j}\left(\begin{array}{l}
j \\
i
\end{array}\right) g_{i}=\sum_{i=0}^{k-1} \sum_{j=i}^{k-1}\left(\begin{array}{l}
j \\
i
\end{array}\right) g_{i}=\sum_{i=0}^{k-1}\left(\begin{array}{c}
k \\
i+1
\end{array}\right) g_{i} .
$$

Changing $i+1 \mapsto i$ we obtain

$$
\left\|\mathcal{M}^{k}\left[F(\alpha)-F\left(\alpha_{0}\right)\right]-\mathcal{M}^{k}\left[F^{\prime}\left(\alpha_{0}\right) r\right]\right\|_{X^{(k)}} \leq \sum_{i=1}^{k}\left(\begin{array}{c}
k \\
i
\end{array}\right)\left(\frac{K}{2}\right)^{i} B^{k-i}\|r\|_{L^{k+i}(\Omega, \mathbb{P} ; Z)}^{k+i} .
$$

The right-hand side is finite if $\alpha \in L^{2 k}(\Omega, \mathbb{P} ; Z)$ and admits the bound as in (4.18) by the binomial theorem.

(2) The assertion (4.19) follows analogously. If $F^{\prime}$ is uniformly bounded, then the estimate in (4.21) can be replaced by

$$
\|\Delta(\omega)\|_{X^{(k)}} \leq k K \theta_{j} B_{*}^{k-1}\|r(\omega)\|_{Z}^{k+1} .
$$

Inserting this into (4.22) yields (4.19). Note that the right-hand side in (4.19) is finite if $\alpha \in L^{k+1}(\Omega, \mathbb{P} ; Z)$, which is a weaker assumption as $\alpha \in L^{2 k}(\Omega, \mathbb{P} ; Z)$ in (1).

The following theorem follows directly from Theorem 3.2 and Lemma 4.7

Theorem 4.8. Suppose that the functional $J$ and its derivatives satisfy the assumptions of Theorem 3.2 . 
(1) If $\mathbb{P}\left\{\left\|\alpha(\omega)-\alpha_{0}\right\|_{Z} \leq \delta\right\}=1$, then

$$
\begin{aligned}
\| \mathcal{M}^{k}\left[S(\alpha)-u_{0}\right]- & \mathcal{M}^{k}\left[S^{\prime}\left(\alpha_{0}\right)\left(\alpha-\alpha_{0}\right)\right] \|_{X(k)} \\
& \leq \delta^{k}\left(\left(\xi_{0}+\delta K / 2\right)^{k}-\xi_{0}^{k}\right)=\mathcal{O}\left(\delta^{k+1}\right) .
\end{aligned}
$$

(2) Suppose $S^{\prime}$ satisfies the local Lipschitz condition

$$
\left\|S^{\prime}(\tilde{\alpha})-S^{\prime}\left(\alpha_{0}\right)\right\|_{Z \rightarrow X} \leq K\left\|\tilde{\alpha}-\alpha_{0}\right\|_{Z} \quad \forall \tilde{\alpha} \in U,
$$

where $U \subseteq Z$ is an open (possibly large or unbounded) set. Assume that $\alpha \in L^{0}(\Omega, \mathbb{P} ; Z)$ is a random field, such that $\mathbb{P}\{\alpha(\omega) \in U\}=1$.

(a) If $\alpha \in L^{2 k}(\Omega, \mathbb{P} ; Z)$, then

$$
\begin{aligned}
\| \mathcal{M}^{k}[S(\alpha) & \left.-u_{0}\right]-\mathcal{M}^{k}\left[S^{\prime}\left(\alpha_{0}\right)\left(\alpha-\alpha_{0}\right)\right] \|_{X^{(k)}} \\
& \leq\left(\left(\xi_{0}+K / 2\right)^{k}-\xi_{0}^{k}\right) \max _{m \in\{k+1, \ldots, 2 k\}}\left\|\alpha-\alpha_{0}\right\|_{L^{m}(\Omega, \mathbb{P} ; Z)}^{m} .
\end{aligned}
$$

(b) If $\alpha \in L^{k+1}(\Omega, \mathbb{P} ; Z)$ and $\xi_{*}:=\sup _{\tilde{\alpha} \in U}\left\|S^{\prime}(\tilde{\alpha})\right\|_{Z \rightarrow X}<\infty$, then

$$
\begin{array}{r}
\left\|\mathcal{M}^{k}\left[S(\alpha)-u_{0}\right]-\mathcal{M}^{k}\left[S^{\prime}\left(\alpha_{0}\right)\left(\alpha-\alpha_{0}\right)\right]\right\|_{X^{(k)}} \\
\leq \frac{k}{2} K \xi_{*}^{k-1}\left\|\alpha-\alpha_{0}\right\|_{L^{k+1}(\Omega, \mathbb{P} ; Z)}^{k+1} .
\end{array}
$$

\section{Sparse tensor Galerkin discretization}

5.1. Formulation of $k$-moment equation. We address efficient sparse tensor discretizations of the abstract first order $k$-th moment equation (4.15), that is,

$$
G_{0}^{(k)} Z^{(k)}=\left[-J_{\alpha}^{\prime}\left(\alpha_{0}, u_{0}\right)\right]^{(k)} \mathcal{M}^{k} r \quad \text { in } X^{(k)} .
$$

We recall that the solution $Z^{(k)} \in X^{(k)}$ of (5.1) approximates $\mathcal{M}^{k}\left[u-u_{0}\right]$ under smallness assumptions on the fluctuation $r(\omega)=\alpha(\omega)-\alpha_{0}$.

Throughout, we assume that $X, Y, Z$ are reflexive Banach spaces and that the sensitivity operator $G_{0} \in \mathcal{L}\left(X, Y^{\prime}\right)$ is boundedly invertible. This implies bounded invertibility of $G_{0}^{(k)} \in \mathcal{L}\left(X^{(k)},\left(Y^{(k)}\right)^{\prime}\right)$. A necessary and sufficient condition for bounded invertibility of $G_{0}$ are the inf-sup conditions: there exists $\gamma>0$ such that

$$
\inf _{0 \neq v \in Y} \sup _{0 \neq w \in X} \frac{\left\langle v, G_{0} w\right\rangle}{\|v\|_{Y}\|w\|_{X}} \geq \gamma, \quad \inf _{0 \neq w \in X} \sup _{0 \neq v \in Y} \frac{\left\langle v, G_{0} w\right\rangle}{\|v\|_{Y}\|w\|_{X}} \geq \gamma .
$$

Here and in what follows, $\langle\cdot, \cdot\rangle$ denotes the $Y \times Y^{\prime}$ duality. To facilitate the discussion, we associate with the sensitivity operator $G_{0}$ the bilinear form

$$
g_{0}(w, v):=\left\langle v, G_{0} w\right\rangle: X \times Y \rightarrow \mathbb{R} .
$$

Based on the general assumptions made so far, the form $g_{0}(\cdot, \cdot): X \times Y \mapsto \mathbb{R}$ is continuous, and satisfies the inf-sup conditions (5.2). An immediate consequence of (5.2) is the following quantification of well-posedness of the $k$-th moment equation (5.1): with the stability constant $\gamma>0$ from (5.2), it holds that

$$
\begin{aligned}
& \inf _{0 \neq v \in Y^{(k)}} \sup _{0 \neq w \in X^{(k)}} \frac{Y^{(k)}\left\langle v,\left[G_{0}\right]^{(k)} w\right\rangle_{X^{(k)}}}{\|v\|_{Y^{(k)}}\|w\|_{X^{(k)}}} \geq \gamma^{k}, \\
& \inf _{0 \neq w \in X^{(k)}} \sup _{0 \neq v \in Y^{(k)}} \frac{Y^{(k)}\left\langle v,\left[G_{0}\right]^{(k)} w\right\rangle_{X^{(k)}}}{\|v\|_{Y^{(k)}}\|w\|_{X^{(k)}}} \geq \gamma^{k} .
\end{aligned}
$$


This shows bounded invertibility of the tensorized operator $G_{0}^{(k)}$ arising in the first order $k$-th moment equation (5.1), however, with condition which deteriorates exponentially with respect to the moment order $k$. We next address efficient discretization of (5.1). To keep technicalities and exposition simple, we shall occasionally impose the (restrictive) assumption of coercivity of $G_{0}$ :

$$
X=Y \quad \text { and } \quad \exists \gamma>0: \forall v \in X: g_{0}(v, v) \geq \gamma\|v\|_{X}^{2} .
$$

Clearly, (5.5) implies (5.2) and (5.4). Note that $X=Y$ does not imply $G_{0}=G_{0}^{*}$.

5.2. Abstract multilevel Galerkin discretization. We present an abstract Galerkin discretization of (5.1). We start with the case $k=1$, and assume the stability condition (5.2). The Galerkin discretization of (5.1) for $k=1$ is based on two dense, nested sequences

$$
\left\{V_{\ell}^{X}\right\}_{\ell=0}^{\infty} \subset X, \quad\left\{V_{\ell}^{Y}\right\}_{\ell=0}^{\infty} \subset Y
$$

of equal and finite dimension, i.e., $N_{\ell}=\operatorname{dim} V_{\ell}^{X}=\operatorname{dim} V_{\ell}^{Y}<\infty$. In the elliptic case (5.5), we have $V_{\ell}^{X}=V_{\ell}^{Y}$ and we write $V_{\ell}=V_{\ell}^{X}=V_{\ell}^{Y}$.

We assume the discrete stability condition: there exists $\bar{\gamma}>0$ and $\ell_{0} \geq 0$ such that for all $\ell \geq \ell_{0}$,

$$
\inf _{0 \neq v \in V_{\ell}^{Y}} \sup _{0 \neq w \in V_{\ell}^{X}} \frac{\left\langle v, G_{0} w\right\rangle}{\|v\|_{Y}\|w\|_{X}} \geq \bar{\gamma}, \quad \inf _{0 \neq w \in V_{\ell}^{X}} \sup _{0 \neq v \in V_{\ell}^{Y}} \frac{\left\langle v, G_{0} w\right\rangle}{\|v\|_{Y}\|w\|_{X}} \geq \bar{\gamma} .
$$

Evidently, in the coercive case (5.5), the discrete stability conditions (5.7) hold with $\ell_{0}=0$ and with $\bar{\gamma}=\gamma$ for any selection of subspaces $V_{\ell}$.

The following result on the stability and the quasioptimality of Galerkin approximations of (5.1) follows from the Lax-Milgram Lemma.

Proposition 5.1. Assume (5.2), (5.7). Then, for any $L \geq \ell_{0}$, and any $k \geq 1$, the tensorized $k$-linear Galerkin equations: find $Z_{L}^{(k)} \in\left[V_{L}^{X}\right]^{(k)}$ satisfying

$$
\left\langle v, G_{0}^{(k)} Z_{L}^{(k)}\right\rangle=(-1)^{k}\left\langle v,\left[J_{\alpha}^{\prime}\left(\alpha_{0}, u_{0}\right)\right]^{(k)} \mathcal{M}^{k} r\right\rangle \quad \forall v \in\left[V_{L}^{Y}\right]^{(k)},
$$

admit a unique solution $Z_{L}^{(k)} \in\left[V_{L}^{X}\right]^{(k)}$ which converges quasioptimally to the unique solution $Z^{(k)} \in X^{(k)}$ of the first order, $k$-th moment equation (5.1). There holds

$$
\left\|Z^{(k)}-Z_{L}^{(k)}\right\|_{X^{(k)}} \leq\left(1+\frac{\left\|G_{0}\right\|^{k}}{\bar{\gamma}^{k}}\right) \inf _{w_{L} \in\left[V_{L}^{X}\right]^{(k)}}\left\|Z^{(k)}-w_{L}\right\|_{X^{(k)}} .
$$

Convergence rates can then be obtained by standard arguments, once regularity of $Z^{(k)}$ in (5.1) is available. To state this in an abstract fashion, we embed $X$ and $Y^{\prime}$ into two scales $\left\{X^{s}\right\}_{s \geq 0}$ and $\left\{Y^{\prime s}\right\}_{s \geq 0}$ of smoothness spaces of Besov type such that

$$
X=X^{0} \supset X^{1} \supset X^{2} \supset \ldots \supset X^{s} \supset \ldots, \quad Y^{\prime}=Y^{\prime 0} \supset Y^{\prime 1} \supset Y^{\prime 2} \supset \ldots \supset Y^{\prime s} \supset \ldots .
$$

Following [24, 26] we assume that the subspaces $V_{\ell}^{X}$ admit the approximation property: for $u \in X^{s}$ and for every $\ell \geq 0$ it holds that

$$
\min _{w_{\ell} \in V_{\ell}^{X}}\left\|u-w_{\ell}\right\|_{X} \lesssim \Phi\left(N_{\ell}, s\right)\|u\|_{X^{s}}
$$

where $\Phi(N, s)$ is a function of $N$ tending to zero as $N \rightarrow \infty$ for every $s>0$ (typically, $\Phi(N, s)=N^{-s / d}$ where $s>0$ denotes a Sobolev, respectively, Besov 
smoothness order and $d \geq 1$ denotes the dimension of the computational domain in which the operator $G_{0}$ is defined).

Based on (5.10) and on the quasioptimality (5.8), we derive convergence rates for the approximation of $Z^{(k)}$ and, by Theorem 4.6, also for $\mathcal{M}^{k}\left[u-u_{0}\right]$.

To state the estimates, based on the smoothness scales (5.9), we introduce for $k \geq 2$ two families of tensorized smoothness scales: the "isotropic" scale $\left[X^{s}\right]_{\text {iso }}^{(k)}$ and the "mixed" scale $\left[X^{s}\right]_{\text {mix }}^{(k)}$ as follows:

$$
\begin{aligned}
{\left[X^{s}\right]_{\text {iso }}^{(k)} } & :=X^{s} \otimes X^{(k-1)} \cap X \otimes X^{s} \otimes X^{(k-2)} \cap \ldots \cap X^{(k-1)} \otimes X^{s}, \\
{\left[X^{s}\right]_{\text {mix }}^{(k)} } & :=X^{s} \otimes \ldots(k-\text { times }) \ldots \otimes X^{s} .
\end{aligned}
$$

Then, for $\ell \geq \ell_{0} \geq 0$, a tensor product argument implies the convergence rate

$$
\left\|Z^{(k)}-Z_{L}^{(k)}\right\|_{X^{(k)}} \lesssim \Phi\left(N_{L}, s\right)\left\|Z^{(k)}\right\|_{\left[X^{s}\right]_{\mathrm{iso}}^{(k)}}
$$

where $\operatorname{dim}\left[V_{L}^{X}\right]^{(k)}=N_{L}^{k}$, i.e., the full tensor product approximation of $Z^{(k)}$ converges at the same rate for all $k \geq 1$ (in particular, therefore, at the rate for the mean field problem $k=1$ ). However, the number of degrees of freedom used in the full tensor approximation increases superlinearly with respect to $N_{L}$ for second and higher order moments. In addition, the convergence estimates (5.12) require only the "isotropic" regularity of order $s$ of $Z^{(k)}$. However, the tensor structure of the operator $G_{0}^{(k)}$ implies, in fact, regularity of order $s>0$ for $Z^{(k)}$ in the mixed smoothness scales $\left[X^{s}\right]_{\mathrm{mix}}^{(k)}$.

Proposition 5.2. Assume (5.2) and that $\Gamma_{0}:=G_{0}^{-1} \in \mathcal{L}\left(Y^{\prime s}, X^{s}\right)$ for some $s>0$. Then $\Gamma_{0}^{(k)}=\left[G_{0}^{(k)}\right]^{-1} \in \mathcal{L}\left(\left[Y^{\prime s}\right]_{\text {mix }}^{(k)},\left[X^{s}\right]_{\text {mix }}^{(k)}\right)$ boundedly.

The mixed regularity $Z^{(k)} \in\left[X^{s}\right]_{\text {mix }}^{(k)}$ is well known to allow for sparse tensor approximation at essentially the rate $\Phi\left(N_{L}, s\right)$, however, from sparse tensor subspaces $\widehat{V_{L}^{X}}(k) \subset\left[V_{L}^{X}\right]^{(k)}$ with substantially fewer degrees of freedom than $\operatorname{dim}\left(\left[V_{L}^{X}\right]^{(k)}\right)=$ $N_{L}^{k}$. Denote for $\ell \geq 0$,

$$
W_{\ell}^{X}:=V_{\ell}^{X} \backslash V_{\ell-1}^{X}, \quad W_{\ell}^{Y}:=V_{\ell}^{Y} \backslash V_{\ell-1}^{Y},
$$

with the convention that $V_{-1}^{X}=V_{-1}^{Y}=\emptyset$. Then the sparse tensor spaces $\widehat{V_{L}^{X}}(k)$, $\widehat{V_{L}^{Y}}(k)$ are defined by

$$
{\widehat{V_{L}^{X}}}^{(k)}:=\bigoplus_{\ell_{1}+\ldots+\ell_{k} \leq L} \bigotimes_{j=1}^{k} W_{\ell_{j}}^{X}, \quad{\widehat{V_{L}^{Y}}}^{(k)}:=\bigoplus_{\ell_{1}+\ldots+\ell_{k} \leq L} \bigotimes_{j=1}^{k} W_{\ell_{j}}^{Y} .
$$

If $N_{\ell}=\operatorname{dim}\left(V_{\ell}^{X}\right)=\mathcal{O}\left(b^{\ell}\right)$ for some basis $b>1$ (in the context of multilevel or wavelet methods considered below, $b=2^{d}$ ), it is easily verified that, as $L \rightarrow \infty$,

$$
\operatorname{dim}\left({\widehat{V_{L}^{X}}}^{(k)}\right)=\operatorname{dim}\left({\widehat{V_{L}^{Y}}}^{(k)}\right)=\mathcal{O}\left(N_{L}\left(\log \left(N_{L}\right)\right)^{k-1}\right) .
$$

To harness the (generically available) additional regularity $Z^{(k)} \in\left[X^{s}\right]_{\text {mix }}^{(k)}$ of the solution of (5.1) and the superior approximation power of the sparse tensor product spaces (5.13) in (nonadaptive) Galerkin approximation schemes, however, the fundamental problem arises in the case $X \neq Y$ that the stability (5.7) in general does not appear to imply a corresponding discrete inf-sup condition on the sparse tensor product spaces $\widehat{V_{L}^{X}}(k), \widehat{V_{L}^{Y}}(k)$. 
Theorem 5.3. Assume (5.2) and that $G_{0}^{-1} \in \mathcal{L}\left(Y^{\prime s}, X^{s}\right)$ for some $s>0$. Assume moreover that for $k>1$ there exists $L_{0}>0$ such that for all $L \geq L_{0}$ the tensorized sensitivity operator $G_{0}^{(k)}$ is stable on the pairs of sparse tensor product spaces ${\widehat{V_{L}^{X}}}^{(k)}$, $\widehat{V_{L}^{Y}}(k)$, i.e., that there exists $\bar{\gamma}(k)>0$ such that

$$
\begin{aligned}
& \inf _{0 \neq v \in \widehat{V}_{L}^{(k)}} \sup _{0 \neq w \in{\widehat{V_{L}^{X}}}^{(k)}} \frac{\left\langle v, G_{0}^{(k)} w\right\rangle}{\|v\|_{Y^{(k)}}\|w\|_{X^{(k)}}} \geq \bar{\gamma}(k), \\
& \inf _{0 \neq w \in{\widehat{V_{L}^{X}}}^{(k)}} \sup _{0 \neq v \in{\widehat{V_{L}^{Y}}}^{(k)}} \frac{\left\langle v, G_{0}^{(k)} w\right\rangle}{\|v\|_{Y^{(k)}}\|w\|_{X^{(k)}}} \geq \bar{\gamma}(k) .
\end{aligned}
$$

Then for the sparse tensor Galerkin approximations: find $\widehat{Z}_{L}^{(k)} \in{\widehat{V_{L}^{X}}}^{(k)}$ such that for all $v \in \widehat{V_{L}^{Y}}(k)$ it holds that

$$
\left\langle v, G_{0}^{(k)} \widehat{Z}_{L}^{(k)}\right\rangle=(-1)^{k}\left\langle v,\left[J_{\alpha}^{\prime}\left(\alpha_{0}, u_{0}\right)\right]^{(k)} \mathcal{M}^{k} r\right\rangle
$$

admits a unique solution $\widehat{Z}_{L}^{(k)} \in{\widehat{V_{L}^{X}}}^{(k)}$. This solution converges quasioptimally, i.e.,

$$
\begin{aligned}
& \left\|Z^{(k)}-\widehat{Z}_{L}^{(k)}\right\|_{X^{(k)}} \lesssim(\bar{\gamma}(k))^{-1} \inf _{w \in \widehat{V}_{L}^{X}(k)}\left\|Z^{(k)}-w\right\|_{X^{(k)}} \\
& \lesssim(\bar{\gamma}(k))^{-1}\left(\log N_{L}\right)^{\frac{k-1}{2}} \Phi\left(N_{L}, s\right)\left\|Z^{(k)}\right\|_{\left[X^{s}\right]_{\text {mix }}^{(k)}}
\end{aligned}
$$

with the number of degrees of freedom bounded by (5.14).

In the remainder of this paper, we illustrate the foregoing abstract theory with an example of the random solution to a nonstationary diffusion equation in a class of stationary, smooth and bounded (this could be weakened) random domains $D \subset \mathbb{R}^{d}$, $d \geq 2$.

\section{Nonstationary heAT EQUATiOn In RANDOM DOMAin}

A broad class of applications of the abstract theory which we developed in sections 25 consists of boundary value problems in random domains. Here, the dependence of solutions on the domain is generically nonlinear, even for linear partial differential equations. The sensitivity operator $G_{0}=J_{\alpha}^{\prime}\left(\alpha_{0}, u_{0}\right)$ is the shape derivative of the random solution evaluated at the nominal domain. The idea to use shape derivatives in first order second moment calculus was first proposed and developed in [14]. The rather well-developed shape calculus as presented, e.g., in [25] (see also [15]) immediately yields broad classes of applications. By the Hadamard formula (e.g. [25, Thm. 2.27]), if the only source of randomness, respectively, uncertainty in the problem of interest is the shape of the domain D, the shape derivative (and, hence, all linearizations of the sensitivity operator $G_{0}$ ) takes the form of a linear boundary value problem without source terms in the nominal domain $D_{0}$. This, and the necessity for sparse tensor spaces in the efficient Galerkin approximation of $k$-point correlation functions mandate boundary integral equation reformulations and their Galerkin discretization by multiresolution schemes on the boundary $\partial D_{0}$ of the nominal domain. Such multiresoltion schemes have the additional advantage that they allow for compression of the Galerkin discretization of $G_{0}$ which, 
in boundary integral formulations, is a nonlocal operator on $\partial D_{0}$. For a stationary diffusion equation, this program was developed in 14. Here, we extend this to time-dependent diffusion in a random domain. We consider here only time independent random perturbations of $D_{0}$ (and thereby avoid having to introduce stochastic processes). We proceed along the lines of [14]: a) shape gradient and determination of $G_{0}$, b) boundary reduction of the first order perturbation problem and c) sparse tensor Galerkin discretization of the boundary reduced problem.

6.1. Shape calculus. As a reference for the shape calculus, we follow 25, Chapter 3.4]. Let $D \subset \mathbb{R}^{d}(d \geq 2)$ denote a bounded domain with smooth boundary $\partial D$ and with exterior unit normal vector field $n$, and with $T>0$ a finite time horizon, $I=(0, T)$ the time interval of interest, and denote by $Q=D \times I$ the space-time cylinder. Of particular relevance in the following will be the "mantle" $\Sigma=\partial D \times I$ of $Q$. In $Q$, we consider the linear, diffusion initial-boundary value problem with either Dirichlet or Neumann boundary conditions. The Neumann problem reads: for given $f \in L^{2}(Q)$ and $y_{0} \in L^{2}(D)$, find $y=y(x, t)$ such that

$$
\begin{array}{cc}
\frac{\partial y}{\partial t}-\Delta y=f & \text { in } Q \\
\gamma_{1} y=\frac{\partial y}{\partial n}=0 & \text { on } \Sigma, \\
y(x, 0)=y_{0}(x) & \text { in } D .
\end{array}
$$

Here and in what follows, $\gamma_{1}$ denotes the (co)normal derivative on the boundary of the nominal domain, $\partial D$.

The weak form of (6.1) is as follows: find $y(t) \in H^{1}(D)$ such that for all $v \in$ $H^{1}(D)$ it holds that

$$
\begin{array}{rr}
\int_{D}\left(v \frac{\partial y}{\partial t}+\nabla y \cdot \nabla v\right) d x=\int_{D} f v d x & \text { a.e. } t \in I, \\
y(0)=y_{0} & \text { in } L^{2}(D) .
\end{array}
$$

It is well known that (6.2) admits a unique weak solution $y \in W\left(I ; H^{1}(D)\right)$ where, for any $H_{0}^{1}(D) \subseteq H \subseteq H^{1}(D)$, the Bochner space $W(I ; H)$ is given by

$$
W(I ; H)=\left\{\phi \in L^{2}(I ; H) \mid \frac{\partial \phi}{\partial t} \in L^{2}\left(I ; H^{\prime}\right)\right\} .
$$

Moreover, $W(I ; H) \subset C^{0}\left(\bar{I} ; L^{2}(D)\right)$ with continuous injection so that the initial condition in (6.2) is well defined.

Analogously, the Dirichlet problem reads: given $f \in L^{2}(Q)$ and $w_{0} \in L^{2}(D)$, find $w=w(x, t)$ such that

$$
\begin{array}{cc}
\frac{\partial w}{\partial t}-\Delta w=f & \text { in } Q, \\
\gamma_{0} w=\left.w\right|_{\Sigma}=0 & \text { on } \Sigma, \\
w(x, 0)=w_{0}(x) & \text { in } D .
\end{array}
$$

In weak form: find $w(t) \in H_{0}^{1}(D)$ such that for all $v \in H_{0}^{1}(D)$ it holds that

$$
\begin{aligned}
\int_{D}\left(v \frac{\partial w}{\partial t}+\nabla w \cdot \nabla v\right) d x=\int_{D} f v d x & \text { a.e. } t \in I \\
w(0)=w_{0} & \text { in } L^{2}(D) .
\end{aligned}
$$


Then (6.4) admits a unique weak solution $w \in W\left(I ; H_{0}^{1}(D)\right)$.

To compute the shape derivatives of $y$ and $w$, we use the speed method: we transport the nominal domain $D$ under a smooth flow and assume that the (deterministic) data $f$ and $y_{0}$ are given in all of $\mathbb{R}^{d}$ (rendering the diffusion problem meaningful on the perturbed domains). Specifically, for $\varepsilon>0$ sufficiently small, given a velocity field $V \in C^{\infty}\left(\mathbb{R}^{d} ; \mathbb{R}^{d}\right)$ and the one-parameter family $D_{s}=$ $T_{s}(V)(D):=\left\{T_{s}(V)(x) \mid x \in D\right\}$ of transported domains under the flow $T_{s}(V)$. For $\varepsilon>0$ sufficiently small, $D_{s}$ is smoothly diffeomorphic to $D_{0}=D$ under $T_{s}(V)$ for $s \in[0, \varepsilon)$.

As shown in [25, Lemma 3.7], the shape derivative $y^{\prime}$ of the solution $y$ of the Neumann problem (6.1) with domain perturbation in direction $V$ is the solution of a nonstationary Neumann problem. With $\gamma_{1}$ denoting the (distributional) conormal derivative on $\Sigma$, it reads: find $y^{\prime}$ such that

$$
\begin{aligned}
& \frac{\partial y^{\prime}}{\partial t}-\Delta y^{\prime}=0 \quad \text { in } \quad Q \\
& \gamma_{1} y^{\prime}=y_{\Sigma}^{\prime}[V \cdot n]:=\gamma_{0}\left(-\frac{\partial y}{\partial t}+f\right)(V \cdot n)+\operatorname{div}_{\partial D}\left((V \cdot n) \nabla_{\partial D} \gamma_{0} y\right) \quad \text { on } \Sigma, \\
& y^{\prime}(x, 0)=0 \quad \text { in } \quad D .
\end{aligned}
$$

Using [25, Proposition 2.68], we obtain the alternative representation

$$
y_{\Sigma}^{\prime}[V \cdot n]=\nabla_{\partial D}(V \cdot n) \cdot \nabla_{\partial D} \gamma_{0} y-(V \cdot n) \frac{\partial^{2} y}{\partial n^{2}} \quad \text { on } \quad \Sigma .
$$

We remark that even for $\partial D \in C^{\infty}$ and smooth $f$ and $y_{0}$, this Cauchy data develops a singularity at $t=0$, unless $f$ and $y_{0}$ satisfy certain compatibility conditions at $\{t=0\} \cap \partial D$ (see, e.g., [1]).

Analogously, the shape derivative $w^{\prime}$ of the solution $w$ of the Dirichlet problem (6.3) solves the following nonhomogeneous, nonstationary Dirichlet problem (see [25, Lemma 3.9]):

$$
\begin{aligned}
& \frac{\partial w^{\prime}}{\partial t}-\Delta w^{\prime}=0 \quad \text { in } \quad Q, \\
& \gamma_{0} w^{\prime}=w_{\Sigma}^{\prime}[V \cdot n]:=-(V \cdot n) \gamma_{1} w \text { on } \Sigma, \\
& w^{\prime}(x, 0)=0 \text { in } D .
\end{aligned}
$$

As already observed by J. Hadamard [13, the Cauchy data $y_{\Sigma}^{\prime}$ and $w_{\Sigma}^{\prime}$ for both shape derivatives, (6.5) and (6.7), depends linearly only on the normal component $\alpha=V \cdot n$ of the perturbation field. Accordingly, we assume in what follows that random domain perturbations are defined by a speed field $V$ of the form $V=$ $\alpha(x, \omega) n$ where $n$ denotes the exterior unit normal to the smooth, nominal domain $D_{0}=D$, and where the scalar function $\alpha$ is a smooth random field on $\partial D$ with zero mean $\alpha_{0}=\mathbb{E}[\alpha]=0$ and known two-point correlation function $\mathcal{M}^{2}[\alpha]=$ $\mathbb{E}\left[\alpha(x, \cdot) \otimes \alpha\left(x^{\prime}, \cdot\right)\right]: \partial D \times \partial D \mapsto \mathbb{R}$. We also observe that both the Dirichlet and Neumann shape derivative problems (6.5) and (6.7) have homogeneous source terms and homogeneous initial conditions.

6.2. Boundary reduction. To develop optimal complexity Galerkin discreizations for the $k$-point correlation functions, we reduce (6.5) and (6.7) in the space 
time cylinder $Q$ to first kind variational boundary integral equations on the lower dimensional cylinder "mantle" $\Sigma$, following 9] (see also 3] and 5]). Denote

$$
E(t, x):=(4 \pi t)^{-d / 2} \exp \left(-|x|^{2} / 4 t\right) \vartheta(t)
$$

the fundamental solution of the heat operator. Here $\vartheta(t)=\frac{1}{2}(1+\operatorname{sign}(t))$ denotes the Heaviside function. By $\kappa_{t_{0}}$ we denote the time-reversal map given by $\kappa_{t_{0}} v(t, x):=v\left(t_{0}-t, x\right)$. Then, for solutions $u(x, t)$ of the homogeneous heat equation there holds the representation formula

$$
u=K_{0}\left(\gamma_{1} u\right)-K_{1}\left(\gamma_{0} u\right) \text { in } Q,
$$

where, for $\left(t_{0}, x_{0}\right) \in Q$ the single layer heat potential $K_{0}$ and the double layer heat potential $K_{1}$ are defined by

$$
K_{0}(\varphi)\left(t_{0}, x_{0}\right):=\left\langle\varphi, \gamma_{0} \kappa_{t_{0}} v\right\rangle, \quad K_{1}(w)\left(t_{0}, x_{0}\right):=\left\langle\gamma_{1} \kappa_{t_{0}} v, w\right\rangle
$$

with $v(t, x)=E\left(t, x_{0}-x\right)$. For the formulation of the variational boundary integral equations, certain anisotropic Sobolev spaces on $\Sigma=\partial D_{0} \times I$ are required: for $r \geq 0$, we denote by

$$
\begin{aligned}
\tilde{H}^{r, r / 2}(\Sigma) & :=L^{2}\left(I ; H^{r}(\partial D)\right) \cap \tilde{H}^{r / 2}\left(I ; L^{2}(\partial D)\right) \\
& \simeq L^{2}(I) \otimes H^{r}(\partial D) \cap \tilde{H}^{r / 2}(I) \otimes L^{2}(\partial D)
\end{aligned}
$$

where $\otimes$ denotes the usual tensor product of separable Hilbert spaces and where $\tilde{H}^{r}(I)=\left\{v \in H^{r}(I) \mid \tilde{v} \in H^{r}(-\infty, T)\right\}$ with $\tilde{v}$ denoting the extension of $v$ by zero to the negative real axis. Note that for $r<1 / 2$ it holds that $\tilde{H}^{r}(I) \simeq H^{r}(I)$. The space $\tilde{H}^{r, r / 2}(Q)$ is defined analogously. To formulate the boundary integral equations, we introduce for $(x, t) \in \Sigma$ the weakly singular and the hypersingular boundary integral operators:

$$
\begin{aligned}
(S \sigma)(x, t) & :=\int_{0}^{t} \int_{\partial D} \sigma(y, \tau)\left(\gamma_{0, y} E\right)(x-y, t-\tau) d s_{y} d \tau, \\
(H \mu)(x, t) & :=-\gamma_{1, x} \int_{0}^{t} \int_{\partial D} \mu(y, \tau)\left(\gamma_{1, y} E\right)(x-y, t-\tau) d s_{y} d \tau .
\end{aligned}
$$

Here, $\gamma_{0, y}$ denotes the trace operator taken with respect to the spatial variable $y$, and $\gamma_{1, x}$ denotes the (co)normal derivative operator taken with respect to the spatial variable $x$. It is by now classical that the operators $S$ and $H$ in (6.11) are continuous and boundedly invertible in anisotropic Sobolev spaces on $\Sigma=\partial D_{0} \times I$ [9. Theorem 3.7, Theorem 4.16]. Specifically, it is shown there that the following operators are bijective for every $|s|<1 / 2$ :

$$
\begin{array}{c:cccc}
S & : & \tilde{H}^{-1 / 2+s,(-1 / 2+s) / 2}(\Sigma) & \rightarrow & \tilde{H}^{1 / 2+s,(1 / 2+s) / 2}(\Sigma), \\
H & : & \tilde{H}^{1 / 2+s,(1 / 2+s) / 2}(\Sigma) & \rightarrow & \tilde{H}^{-1 / 2+s,(-1 / 2+s) / 2}(\Sigma) .
\end{array}
$$

The Sobolev range of bijectivity $|s|<1 / 2$ can be enlarged to arbitrary $s>0$ for smooth $\partial D \in C^{\infty}$, according to [9, Proposition 4.3]. The boundary reduction of the shape derivative problems (6.7), 6.5) is now straightforward (see [9, Corollary $3.16 \mathrm{c}), 3.17 \mathrm{~d})]$ ): the unique variational solution $w^{\prime}$ of the Dirichlet problem (6.7) can be represented as

$$
w^{\prime}=K_{0} \psi, \quad \text { where } \quad S \psi=w_{\Sigma}^{\prime}[V \cdot n],
$$

and the unique solution of the Neumann problem as

$$
y^{\prime}=K_{1} \zeta, \quad \text { where } \quad H \zeta=-y_{\Sigma}^{\prime}[V \cdot n]
$$


with $w_{\Sigma}^{\prime}$ and $y_{\Sigma}^{\prime}$ as in (6.7), (6.1) respectively. The unique solvability of the boundary integral equations (6.13) and (6.14) is ensured by the following coercivity properties of the weakly singular boundary integral operator $S$ and of the hypersingular boundary integral operator $H$ (see [9, Corollary 3.13]): there exists a constant $\gamma>0$ such that

$$
\forall \psi \in H^{-1 / 2,-1 / 4}(\Sigma): \quad\langle\psi, S \psi\rangle \geq \gamma\|\psi\|_{H^{-1 / 2,-1 / 4}(\Sigma)}^{2},
$$

and, with $\langle\cdot, \cdot\rangle$ denoting the corresponding duality pairings,

$$
\forall \zeta \in H^{1 / 2,1 / 4}(\Sigma): \quad\langle\zeta, H \zeta\rangle \geq \gamma\|\zeta\|_{H^{1 / 2,1 / 4}(\Sigma)}^{2} .
$$

For a given, smooth velocity field $V \in C^{\infty}\left(\partial D_{0} ; \mathbb{R}^{3}\right)$, the unique solution $w^{\prime}$ of the Dirichlet problem (6.7) is given by $w^{\prime}=K_{0} \psi$ where the unknown surface density $\psi$ is the solution of the variational first kind boundary integral equation: find

$$
\text { (6.17) } \psi \in H^{-1 / 2,-1 / 4}(\Sigma): \quad\langle\bar{\psi}, S \psi\rangle=\left\langle\bar{\psi}, w_{\Sigma}^{\prime}[V \cdot n]\right\rangle \quad \forall \bar{\psi} \in H^{-1 / 2,-1 / 4}(\Sigma) .
$$

By (6.15), the BIE (6.17) admits a unique solution for every $w_{\Sigma}^{\prime}[V \cdot n] \in H^{1 / 2,1 / 4}(\Sigma)$. Moreover, by $\left[9\right.$ if $w_{\Sigma}^{\prime}[V \cdot n] \in H^{1 / 2+s / 2,1 / 4+s / 4}(\Sigma)$ with $s>0$ this solution belongs to $H^{-1 / 2+s / 2,-1 / 4+s / 4}(\Sigma)$.

Analogously, the variational formulation of (6.14) reads: find

$$
\zeta \in H^{1 / 2,1 / 4}(\Sigma): \quad\langle\bar{\zeta}, H \zeta\rangle=-\left\langle\bar{\zeta}, y_{\Sigma}^{\prime}[V \cdot n]\right\rangle \quad \forall \bar{\zeta} \in H^{1 / 2,1 / 4}(\Sigma) .
$$

By (6.16), for every $y_{\Sigma}^{\prime}[V \cdot n] \in H^{-1 / 2,-1 / 4}(\Sigma)$, the BIE (6.18) admits a unique solution $\zeta \in H^{1 / 2,1 / 4}(\Sigma)$ and if $y_{\Sigma}^{\prime}[V \cdot n] \in H^{-1 / 2+s / 2,-1 / 4+s / 4}(\Sigma)$ for $s>0$ we have $\zeta \in H^{1 / 2+s / 2,1 / 4+s / 4}(\Sigma)$.

6.3. Sparse space-time Galerkin discretization. The coercivity (6.15) and (6.16) of the first kind boundary integral operators $S$ and $H$ imply stability and quasioptimality of Galerkin discretizations of the BIEs (6.17), 66.18) (e.g. 20]) for any closed subspace $V_{h}$ of $V=H^{ \pm 1 / 2, \pm 1 / 4}(\Sigma)$. For brevity of exposition, we develop the sparse tensor discretization only for the Galerkin BEM for the BIE (6.18) of the Neumann problem (6.1). Analogous results (and proof) hold for BIE (6.17) for the Dirichlet problem (6.7). We refer to [8] for further details. To connect to the abstract Galerkin discretization framework in Section 5.2 we note that here $X=Y=H^{1 / 2,1 / 4}(\Sigma)=: V$ and, due to the ellipticity (6.16), for any closed subspace $V_{h} \subset H^{1 / 2,1 / 4}(\Sigma)$, the Galerkin equations

$$
\zeta_{h} \in V_{h}: \quad\left\langle\bar{\zeta}_{h}, H \zeta_{h}\right\rangle=-\left\langle\bar{\zeta}_{h}, y_{\Sigma}^{\prime}[V \cdot n]\right\rangle \quad \forall \bar{\zeta}_{h} \in V_{h} .
$$

are uniquely solvable and the Galerkin solutions $\zeta_{h} \in V_{h}$ are quasioptimal, i.e.,

$$
\left\|\zeta-\zeta_{h}\right\|_{H^{1 / 2,1 / 4}(\Sigma)} \leq \gamma^{-1} \inf _{v_{h} \in V_{h}}\left\|\zeta-v_{h}\right\|_{H^{1 / 2,1 / 4}(\Sigma)} .
$$

Key to efficient Galerkin discretizations is therefore the proper choice of $V_{h}$. Owing to the (Cartesian) product structure of $\partial D \times I$, finite dimensional subspaces $V_{h}$ can be constructed from tensor products of hierarchic multilevel subspaces of piecewise polynomials of degree $p_{x} \geq 1$, continuous in space, and of degree $p_{t} \geq 0$, possibly discontinuous in time. For simplicity of presentation we consider here only the case $p_{x}=p_{t}=: p$ and refer to [8] for the general analysis. Our generic notation for these spaces is as follows:

$$
V_{0}^{x} \subset V_{1}^{x} \subset \ldots \subset V_{\ell_{x}}^{x} \subset L^{2}(\partial D), \quad V_{0}^{t} \subset V_{1}^{t} \subset \ldots \subset V_{\ell_{t}}^{t} \subset L^{2}(I)
$$


Here, $\ell_{x}, \ell_{t}$ denote "levels of mesh refinement", and we have in mind piecewise polynomial functions on uniformly refined meshes of widths $2^{-\ell_{x}}$ and $2^{-\ell_{t}}$, respectively.

The starting point for sparse space-time tensor Galerkin discretizations of the BIEs (6.17) and in (6.18) are multilevel decompositions of the hierarchies $\left\{V_{\ell}^{x}\right\}_{\ell=0}^{\infty}$ and $\left\{V_{\ell}^{t}\right\}_{\ell=0}^{\infty}$, respectively: for every level $L \geq 1$, we have

$$
V_{L}^{x}=W_{0}^{x} \oplus W_{1}^{x} \oplus \ldots \oplus W_{L}^{x}, \quad V_{L}^{t}=W_{0}^{t} \oplus W_{1}^{t} \oplus \ldots \oplus W_{L}^{t}
$$

where we assume that we are explicitly given bases for the "detail" spaces $W_{\ell}^{x}$ and $W_{\ell}^{t}$ (such bases are explicitly available, for example, in terms of hierarchic Finite Element bases or in terms of spline-wavelet bases as e.g. developed in [5]).

We build the family $\left\{\hat{V}_{L}\right\}_{L=0}^{\infty} \subset H^{1 / 2,1 / 4}(\Sigma)$ of sparse tensor subspaces from the hierarchies (6.21) by

$$
\hat{V}_{L}:=\bigoplus_{\ell_{x}+\ell_{t} \leq L} W_{\ell_{x}}^{x} \otimes W_{\ell_{t}}^{t} \subset V=H^{1 / 2,1 / 4}(\Sigma), \quad L=1,2, \ldots
$$

The corresponding (unique, by (6.16)) Galerkin solution of (6.19) with the subspace $\hat{V}_{L}$ defined in (6.22) will be denoted by $\hat{\zeta}_{L}$. By the quasioptimality (6.20) and our regularity assumptions, the rate of convergence will be determined by the consistency of the family $\left\{\hat{V}_{\ell}\right\}_{\ell \geq 1}$ for smooth $\zeta$, i.e., we assume

$$
\zeta \in H^{1 / 2+s / 2,1 / 4+s / 4}\left(\partial D_{0} \times I\right) \text { for all } s \geq 0 .
$$

In order to bound the convergence rates, we use that the anisotropic spaces (6.10) are intersection spaces and, therefore, are equipped with the sum-norms

$$
\left\|\zeta-\hat{\zeta}_{L}\right\|_{H^{1 / 2,1 / 4}(\Sigma)} \simeq\left\|\zeta-\hat{\zeta}_{L}\right\|_{H_{\text {mix }}^{1 / 2,0}(\Sigma)}+\left\|\zeta-\hat{\zeta}_{L}\right\|_{H_{\text {mix }}^{0,1 / 4}(\Sigma)}
$$

where the spaces $H_{\text {mix }}^{s, t}(\Sigma)$ of "square integrable, mixed highest derivative" are as in e.g. [1] defined by the tensor products: $H_{\text {mix }}^{s, t}(\Sigma):=H^{s}\left(\partial D_{0}\right) \otimes H^{t}(I)$. We may therefore apply [11, Theorem 7.1] (with $\sigma=1, q_{1}=1 / 2, q_{2}=0, r_{1}=r_{2}=p+1$ ) to obtain that for $\zeta \in H_{\text {mix }}^{s_{x}, s_{t}}(\Sigma)$ there exists a (quasi)interpolant $\hat{\zeta}_{L} \in \hat{V}_{L}$ such that for every $1 / 2<s_{x} \leq p+1,1 / 4<s_{t} \leq p+1$ it holds that

$$
\left\|\zeta-\hat{\zeta}_{L}\right\|_{H_{\text {mix }}^{1 / 2,0}(\Sigma)} \lesssim 2^{-L \min \left\{s_{x}-1 / 2, s_{t}\right\}}\|\zeta\|_{H_{\text {mix }}^{s_{x}, s_{t}}(\Sigma)}
$$

and

$$
\left\|\zeta-\hat{\zeta}_{L}\right\|_{H_{\text {mix }}^{0,1 / 4}(\Sigma)} \lesssim 2^{-L \min \left\{s_{x}, s_{t}-1 / 4\right\}}\|\zeta\|_{H_{\text {mix }}^{s_{x}, s_{t}}(\Sigma)}
$$

which implies that for $1 / 2<s_{x} \leq p+1,1 / 4<s_{t} \leq p+1$,

$$
\left\|\zeta-\hat{\zeta}_{L}\right\|_{H^{1 / 2,1 / 4}(\Sigma)} \lesssim 2^{-L \min \left\{s_{x}-1 / 2, s_{t}-1 / 4\right\}}\|\zeta\|_{H_{\text {mix }}^{s_{x}, s_{t}}(\Sigma)},
$$

while, for $d=3$ ([11, Theorem 4.1]), as $L \rightarrow \infty$ we have

$$
\hat{N}_{L}:=\operatorname{dim}\left(\hat{V}_{L}\right) \lesssim 2^{L(d-1)},
$$

i.e., the number of degrees of freedom of the sparse tensor product space $\hat{V}_{L}$ on $\Sigma$ scales, as $L \rightarrow \infty$, as $\mathcal{O}\left(h_{x}^{-(d-1)}\right)$, i.e., of a boundary element spaces on $\partial D_{0}$ with uniform mesh of meshwidth $h_{x}$ (relation (6.25) remains valid also for $d=2$, 
with a logarithmic factor). We sum up these results in the (slightly conservative) asymptotic bounds for the Galerkin discretization error

$$
\begin{aligned}
\left\|\zeta-\hat{\zeta}_{L}\right\|_{H^{1 / 2,1 / 4}(\Sigma)} & \lesssim 2^{-L(p+1 / 2)}\|\zeta\|_{H_{\operatorname{mix}}^{p+1, p+\frac{3}{4}}(\Sigma)} \\
& \lesssim \hat{N}_{L}^{-(p+1 / 2) /(d-1)}\|\zeta\|_{H^{3 p+\frac{5}{2}, \frac{3}{2} p+\frac{5}{4}(\Sigma)}}
\end{aligned}
$$

which is, again, the convergence rate afforded by a Galerkin boundary element discretization of a hypersingular boundary integral equation on a domain $D_{0} \subset \mathbb{R}^{d}$ by continuous, piecewise polynomial boundary elements of degree $p \geq 1$ on $\partial D_{0}$. Given the rather high smoothness of the heat kernel $E(t, x)$ in (6.8), by choosing wavelet bases for the detail spaces $W_{\ell}^{x}$ and $W_{\ell}^{t}$ in the decompositions (6.21) which have a sufficiently high number of vanishing moments, the stiffness matrices of the boundary integral operators $H$ (and of $S$ ) can be compressed to $\mathcal{O}\left(\hat{N}_{L}\right)$ nonvanishing entries without compromising the convergence rate (6.26). In addition, with the wavelets being Riesz bases in the spaces $L^{2}(I)$ and $L^{2}\left(\partial D_{0}\right)$, respectively, and, properly scaled, also in $H^{1 / 4}(I)$ and in $H^{1 / 2}\left(\partial D_{0}\right)$, the matrices of the Galerkin discretized operator $S$ and $H$ have condition numbers which are uniformly bounded with respect to $L$, ensuring mesh independent covergence rates of iterative solvers. In particular, if

$$
\left\{\phi_{\lambda}^{x}: \lambda \in \nabla_{x}\right\} \subset H^{1 / 2}\left(\partial D_{0}\right), \quad\left\{\phi_{\mu}^{t}: \mu \in \nabla_{t}\right\} \subset H^{1 / 4}(I)
$$

are collections of functions being normalized Riesz bases for $L^{2}\left(\partial D_{0}\right)$ and $L^{2}(I)$, respectively, then the collection

$$
\left\{(x, t) \mapsto \frac{\phi_{\lambda}^{x}(x) \phi_{\mu}^{t}(t)}{\sqrt{\left\|\phi_{\lambda}^{x}\right\|_{H^{1 / 2}\left(\partial D_{0}\right)}^{2}+\left\|\phi_{\mu}^{t}\right\|_{H^{1 / 4}(I)}^{2}}}:(\lambda, \mu) \in \nabla_{x} \times \nabla_{t}\right\}
$$

is a Riesz basis for $H^{1 / 2,1 / 4}(\Sigma)$ (see, e.g., 12, 23, 22, for details).

In [8] we show that the a priori estimate (6.26) can be slightly improved if the isotropic sparse tensor product space $\hat{V}_{L}$ in (6.22) is replaced by the anisotropic sparse tensor product construction

$$
\bigoplus_{\ell_{x} \sigma+\ell_{t} / \sigma \leq L} W_{\ell_{x}}^{x} \otimes W_{\ell_{t}}^{t}
$$

with a suitable $0<\sigma<\infty$; see also [1]. In particular, the choice $\sigma=\sqrt{d-1}$ provides a better convergence estimate. We refer to [8] for the detailed analysis.

Naturally, $\hat{\zeta}_{L}$ is only an approximate density on $\Sigma$. Information on the solution inside the space-time cylinder $Q$ must be extracted by inserting $\hat{\zeta}_{L}$ into the representation formula (6.14). This, in fact, allows for superconvergent approximations of $u(x, t)$ as follows by an Aubin-Nitsche duality type argument for the Galerkin BIE (6.18); see 21] for details on this in the elliptic setting.

6.4. Sparse space-time tensor Galerkin discretization for the $k$-th moment equation. Tensorization of (6.13), 6.14) yields

$$
\mathcal{M}^{k}\left[w^{\prime}\right]=K_{0}^{(k)} \mathcal{M}^{k}[\psi], \quad \text { where } \quad S^{(k)} \mathcal{M}^{k}[\psi]=\left(w_{\Sigma}^{\prime}\right)^{(k)} \mathcal{M}^{k}[V \cdot n]
$$

and

$$
\mathcal{M}^{k}\left[y^{\prime}\right]=K_{1}^{(k)} \mathcal{M}^{k}[\zeta], \quad \text { where } \quad H^{(k)} \mathcal{M}^{k}[\zeta]=\left(-y_{\Sigma}^{\prime}\right)^{(k)} \mathcal{M}^{k}[V \cdot n]
$$


As discussed in Section [5, the coercivity (6.15), (6.16) of the boundary integral operators $S$ and $H$ implies well-posedness of (6.28) and (6.29) and stability and quasioptimality of Galerkin discretizations of the $k$-th moment BIE. We again work out the details for the Neumann problem (6.1) and (6.29). Analogous results also hold for (6.7) and (6.28).

Suppose $\hat{V}_{L}$ is a family of sparse tensor subspaces defined in (6.22). We introduce

$$
\widehat{\hat{V}}_{L}^{(k)}:=\bigoplus_{L_{1}+\ldots+L_{k} \leq L} \bigotimes_{j=1}^{k} \hat{W}_{L_{j}} \subset V^{(k)}, \quad V=H^{1 / 2,1 / 4}(\Sigma)
$$

with the "detail" spaces

$$
\hat{W}_{L_{j}}:=\hat{V}_{L_{j}} \backslash \hat{V}_{L_{j}-1}=\bigoplus_{\ell_{x}+\ell_{t}=L_{j}} W_{\ell_{x}}^{x} \otimes W_{\ell_{t}}^{t} .
$$

Due to (6.16) the Galerkin equations

$$
\zeta_{L}^{(k)} \in{\widehat{\hat{V}_{L}}}^{(k)}: \quad\left\langle v, H^{(k)} \zeta_{L}^{(k)}\right\rangle=(-1)^{k}\left\langle v,\left(y_{\Sigma}^{\prime}\right)^{(k)}[V \cdot n]\right\rangle \quad \forall v \in{\widehat{\hat{V}_{L}}}^{(k)}
$$

are uniquely solvable and the Galerkin solutions $\zeta_{L}^{(k)} \in{\widehat{\hat{V}_{L}}}^{(k)}$ are quasioptimal, i.e., for $d=3$ (6.26) yields

$$
\begin{aligned}
\left\|\mathcal{M}^{k}[\zeta]-\zeta_{L}^{(k)}\right\|_{\left[H^{1 / 2,1 / 4}(\Sigma)\right](k)} & \leq \gamma^{-k} \inf _{v_{L} \in \hat{\hat{V}}_{L}(k)}\left\|\mathcal{M}^{k}[\zeta]-v_{L}\right\|_{\left[H^{1 / 2,1 / 4}(\Sigma)\right]^{(k)}} \\
& \lesssim \gamma^{-k} L^{\frac{k-1}{2}} 2^{-L(p+1 / 2)}\left\|\mathcal{M}^{k}[\zeta]\right\|_{\left[H^{r, r / 2}(\Sigma)\right]_{\text {mix }}^{(k)}}
\end{aligned}
$$

where $r=3 p+\frac{5}{2}$. As $L \rightarrow \infty$, the cardinality of $\widehat{\hat{V}}_{L}^{(k)}$ scales as

$$
\operatorname{dim}\left(\widehat{\hat{V}}_{L}^{(k)}\right) \sim \log \left(\hat{N}_{L}\right)^{k-1} \hat{N}_{L} \lesssim L^{k-1} 2^{L(d-1)},
$$

i.e., (up to a logarithmic factor) as the cardinality of the boundary element space on $\partial D_{0}$ with the uniform meshwidth $h_{x} \sim 2^{-L}$.

The approximation $\mathcal{Y}_{L}^{\prime}$ to $\mathcal{M}^{k}\left[y^{\prime}\right]$ is defined via the representation formula

$$
\mathcal{Y}_{L}^{\prime}:=K_{1}^{(k)} \zeta_{L}^{(k)} \text {. }
$$

The mapping $K_{1}: H^{1 / 2,1 / 4}(\Sigma) \rightarrow \tilde{H}^{1,1 / 2}(Q)$ is continuous, thus

$$
\left\|\mathcal{M}^{k}\left[y^{\prime}\right]-\mathcal{Y}_{L}^{\prime}\right\|_{\left[H^{1,1 / 2}(Q)\right]_{\text {mix }}^{(k)}} \leq\left\|K_{1}\right\|^{k}\left\|\mathcal{M}^{k}[\zeta]-\zeta_{L}^{(k)}\right\|_{\left[H^{1 / 2,1 / 4}(\Sigma)\right]_{\text {mix }}^{(k)}} .
$$

Finally, we utilize (4.24) from Theorem 4.8 and obtain by the triangle inequality the error estimate for the $k$-th moment:

$$
\begin{aligned}
& \left\|\mathcal{M}^{k}\left[\frac{y_{s}-y_{0}}{s}\right]-\mathcal{Y}_{L}^{\prime}\right\|_{\left[H^{1,1 / 2}(Q)\right]_{\text {mix }}^{(k)}} \\
& \quad \leq\left\|\mathcal{M}^{k}\left[\frac{y_{s}-y_{0}}{s}\right]-\mathcal{M}^{k}\left[y^{\prime}\right]\right\|_{\left[H^{1,1 / 2}(Q)\right]_{\text {mix }}^{(k)}}+\left\|\mathcal{M}^{k}\left[y^{\prime}\right]-\mathcal{Y}_{L}^{\prime}\right\|_{\left[H^{1,1 / 2}(Q)\right]_{\text {mix }}^{(k)}} \\
& \quad \leq(\delta / s)^{k}\left(\left(\xi_{0}+\delta K / 2\right)^{k}-\xi_{0}^{k}\right)+C\left\|K_{1}\right\| L^{\frac{k-1}{2}} 2^{-L(p+1 / 2)}\left\|\mathcal{M}^{k}[\zeta]\right\|_{\left[H^{r, r / 2}(\Sigma)\right]_{\text {mix }}^{(k)}}
\end{aligned}
$$

where $y_{s}$ is the exact solution of (6.1) in the perturbed spatial domain $D_{s}=$ $T_{s}(V)(D)$; see Subsection 6.1. The constants $\xi_{0}, K$ and $\delta$ were explicitly defined in Section 4 and depend on the size of the random boundary perturbation $\alpha$. 
Remark 6.1. The bound 6.35) suggests the level $L$ of the Finite Element discretization $\widehat{\hat{V}}_{L}{ }^{(k)}$ should be chosen so as to balance the linearization error represented by the first term in the right-hand side of 6.35) $L=L^{o p t}$. For $L>L^{\text {opt }}$ the linearization error dominates the total error in (6.35) and further refinement will not provide a better approximation of the $k$-th moment $\mathcal{M}^{k}\left[\frac{y_{s}-y_{0}}{s}\right]$.

The linearization error depends on the parameters $\xi_{0}, K$ and $\delta$. In the next subsection we exemplify the abstract linearization error bounds by giving explicit values of these constants for the parabolic model problem under consideration.

6.5. Bounds on the linearization error. We recall definitions (3.1), (3.19) and Theorem 4.8(1) of the constants $\xi_{0}, K$ and $\delta$. For the parabolic model problem (6.1), the spaces $X, Y, Z$ from Section 4 are given by

$$
\begin{aligned}
& X:=\tilde{H}^{1, \frac{1}{2}}\left(Q, \frac{\partial}{\partial t}-\Delta\right):=\left\{u \in \tilde{H}^{1, \frac{1}{2}}(Q) \mid\left(\frac{\partial}{\partial t}-\Delta\right) u \in L^{2}(Q)\right\}, \\
& Y:=\tilde{H}^{1, \frac{1}{2}}(Q), \quad Z:=C^{k}\left(\mathbb{R}^{d}\right)
\end{aligned}
$$

with $k \geq 1$ being sufficiently large. Note that $\left\{u \in W\left(I, H^{1}(D)\right) \mid u(x, 0)=0\right\} \subset$ $\tilde{H}^{1, \frac{1}{2}}(Q)$ by interpolation. In this setting we have

$$
\xi_{0}=\left\|\Gamma_{0} J_{\alpha}^{\prime}\left(\alpha_{0}, y_{0}\right)\right\|_{C^{k}\left(\mathbb{R}^{d}\right) \rightarrow H^{1, \frac{1}{2}}(Q)}=\sup _{\alpha \in C^{k}\left(\mathbb{R}^{d}\right)} \frac{\left\|y_{0}^{\prime}(\alpha)\right\|_{H^{1, \frac{1}{2}}(Q)}}{\|\alpha\|_{C^{k}\left(\mathbb{R}^{d}\right)}}
$$

and

$$
\left\|y^{\prime}-y_{0}^{\prime}\right\|_{C^{k}\left(\mathbb{R}^{d}\right) \rightarrow H^{1, \frac{1}{2}}(Q)} \leq K\left\|\alpha-\alpha_{0}\right\|_{C^{k}\left(\mathbb{R}^{d}\right)} .
$$

To avoid ambiguity we denote here by $y_{0}^{\prime}$ the solution of (6.1) in the nominal domain $D=D_{0}$ corresponding to the zero perturbation $\alpha_{0}=0$ whereas $y^{\prime}$ the solution of (6.1) on the perturbed domain $D_{s}=T_{s}(V)(D)$ corresponding to the perturbation parameter $\alpha$. First, we derive an explicit bound for $\xi_{0}$. By continuity of $K_{1}$ and coercivity (6.16) of $H$ we obtain

$$
\left\|y^{\prime}\right\|_{H^{1,1 / 2}(Q)} \leq\left\|K_{1}\right\|\|\zeta\|_{H^{\frac{1}{2}, \frac{1}{4}(\Sigma)}} \leq \frac{\left\|K_{1}\right\|}{\gamma}\left\|y_{\Sigma}^{\prime}[\alpha]\right\|_{H^{-\frac{1}{2},-\frac{1}{4}(\Sigma)}}
$$

and by (6.6)

$$
\left\|y_{\Sigma}^{\prime}[\alpha]\right\|_{H^{-\frac{1}{2},-\frac{1}{4}(\Sigma)}} \leq\|\alpha\|_{W^{1, \infty}(\partial D)}\left(\left\|\nabla_{\partial D} \gamma_{0} y\right\|_{H^{-\frac{1}{2},-\frac{1}{4}(\Sigma)}}+\left\|\frac{\partial^{2} y}{\partial n^{2}}\right\|_{H^{-\frac{1}{2},-\frac{1}{4}(\Sigma)}}\right) .
$$

Thus

$$
\xi_{0}=\frac{\left\|K_{1}\right\|}{\gamma}\left(\left\|\nabla_{\partial D} \gamma_{0} y\right\|_{H^{-\frac{1}{2},-\frac{1}{4}(\Sigma)}}+\left\|\frac{\partial^{2} y}{\partial n^{2}}\right\|_{H^{-\frac{1}{2},-\frac{1}{4}(\Sigma)}}\right)
$$

provided the nominal solution $y$ is sufficiently regular.

Next, we identify the local Lipschitz constant $K$. If the second shape derivative (the "shape-Hessian") of $y$ exists at the nominal domain, then

$$
K=\sup _{\|\alpha\|_{C^{k}\left(\mathbb{R}^{d}\right)} \leq \delta}\left\|y^{\prime \prime}(\alpha)\right\|_{C^{k}\left(\mathbb{R}^{d}\right) \times C^{k}\left(\mathbb{R}^{d}\right) \rightarrow H^{1, \frac{1}{2}}(Q)} .
$$


The second shape derivative of $y^{\prime \prime}$ can be identified as a solution of the following problem:

$$
\begin{array}{ll}
\frac{\partial y^{\prime \prime}}{\partial t}-\Delta y=0 & \text { in } Q_{s}, \\
\frac{\partial y^{\prime \prime}}{\partial n}=y_{\Sigma}^{\prime \prime}[V \cdot n, W \cdot n] & \text { on } \Sigma_{s}, \\
y^{\prime \prime}(x, 0)=0 & \text { in } D_{s},
\end{array}
$$

where

$$
\begin{aligned}
y_{\Sigma}^{\prime \prime}[\alpha, \beta]=-\alpha \beta \frac{\partial^{3} y}{\partial n^{3}} & +\nabla_{\partial D_{s}} \beta \cdot \nabla_{\partial D_{s}} y^{\prime}[\alpha]-\beta \frac{\partial^{2} y^{\prime}[\alpha]}{\partial n_{s}^{2}} \\
& +\nabla_{\partial D_{s}} \alpha \cdot \nabla_{\partial D_{s}} y^{\prime}[\beta]-\alpha \frac{\partial^{2} y^{\prime}[\beta]}{\partial n_{s}^{2}} .
\end{aligned}
$$

Similarly as above, we obtain

$$
\begin{aligned}
K= & \frac{\left\|K_{1}\right\|}{\gamma}\left(\left\|\nabla_{\partial D_{s}} y^{\prime}[\cdot]\right\|_{C^{k}\left(\mathbb{R}^{d}\right) \rightarrow H^{-\frac{1}{2},-\frac{1}{4}}(\Sigma)}\right. \\
& \left.+\left\|\frac{\partial^{2} y^{\prime}[\cdot]}{\partial n_{s}^{2}}\right\|_{C^{k}\left(\mathbb{R}^{d}\right) \rightarrow H^{-\frac{1}{2},-\frac{1}{4}(\Sigma)}}+\left\|\frac{\partial^{3} y}{\partial n_{s}^{3}}\right\|_{H^{-\frac{1}{2},-\frac{1}{4}(\Sigma)}}\right) .
\end{aligned}
$$

The value of $k$ in (6.36) and (6.40) must be chosen so that the operator norms in the right-hand side of (6.40) remain bounded. By (6.14) this task reduces to the study of the mapping properties of nonclassical boundary integral operators

$$
\nabla_{\partial D} K_{1} \quad \text { and } \quad \frac{\partial^{2}}{\partial n^{2}} K_{1}
$$

which is beyond the scope of the present paper; see [9] for more details.

\section{CONCLUding REMARKS}

We provide a first order, $k$-th moment analysis for Fréchet-differentiable nonlinear operator equations with random input data. In Section 3 we studied the solvability of a general nonlinear parametric equation in Banach spaces for varying parameters and obtained estimates on the size of the neighborhoods where the solution operator is well defined (Theorem 3.1. Theorem 3.2). These quantitative results appear to be new and provide a precision of the classical Implicit Function Theorem.

In Section 4 this result has been applied to abstract nonlinear equations in Banach spaces with random input parameters. We established precise nonasymptotic error bounds with explicit constants for the magnitude of the linearization error for the $k$-th moment equation (see Theorem 4.8 being a generalization of the asymptotic estimate in Theorem 4.6).

In Section 5 we presented the numerical analysis of an abstract multilevel Galerkin discretization for the linearized $k$-th moment equations. As a result of Theorem 5.3, the $k$-th moment equation in the $k$-fold cartesian product of the physical domain can be solved by Galerkin approximation at essentially the same cost versus accuracy as a single linearized problem in the original nontensorized domain.

In Section 6 we applied the general methodology to model nonstationary nonhomogeneous parabolic equations in randomly perturbed domains. By means of 
Shape Calculus for the underlying partial differential equations we obtain a formulation for the shape derivative of the solution which does not contain source terms. It can, therefore, be efficiently treated numerically via boundary reduction to strongly positive first kind boundary integral equations on the boundary of the space-time cylinder.

We introduce sparse tensor product Galerkin discretizations of these boundary integral equations. Sparsity enters here on two levels: first, the space and time discretizations are performed by sparse tensorization, thereby compressing the Galerkin approximation unknown surface density to a format with complexity equal to that of a boundary integral equation of a stationary problem.

Second, the hierarchic multilevel space-time tensor basis allows us to exploit the mixed regularity of the $k$-point correlations functions. This leads to a priori error estimates for the resulting nonstationary $k$-th moment equation which equal, up to logarithmic terms, those for the mean field of a stationary diffusion problem on the boundary of the physical domain. In particular, we solve the second moment problem for a three-dimensional nonstationary heat conduction, being an eight-dimensional problem, with the same error versus number of unknowns as a stationary diffusion problem in a two-dimensional domain. Finally, we derive a bound for the total error (6.35) with explicit bounds for the linearization and the discretization errors.

We solved the problems on the boundary of the space-time cylinder using boundary reduction via first kind boundary integral equations. Their stability in parabolic trace spaces is a result of the coercivity of the underlying integral operators. An alternative approach using boundary integral equations of the second kind is also possible.

\section{REFERENCES}

1. Herbert Amann, Linear and quasilinear parabolic problems. Vol. I, Monographs in Mathematics, vol. 89, Birkhäuser Boston, Inc., Boston, MA, 1995, Abstract linear theory. MR 1345385 (96g:34088)

2. Antonio Ambrosetti and Giovanni Prodi, A primer of nonlinear analysis, Cambridge Studies in Advanced Mathematics, vol. 34, Cambridge University Press, Cambridge, 1993. MR.1225101 (94f:58016)

3. Douglas N. Arnold and Patrick J. Noon, Coercivity of the single layer heat potential, J. Comput. Math. 7 (1989), no. 2, 100-104, China-US Seminar on Boundary Integral and Boundary Element Methods in Physics and Engineering (Xi'an, 1987-88). MR1016829 (90k:65186)

4. Melvin S. Berger, Nonlinearity and functional analysis, Academic Press [Harcourt Brace Jovanovich Publishers], New York, 1977, Lectures on nonlinear problems in mathematical analysis, Pure and Applied Mathematics. MR0488101 (58:7671)

5. Christian Bourgeois and Serge Nicaise, Prewavelet analysis of the heat equation, Numer. Math. 87 (2001), no. 3, 407-434. MR1811643(2001h:65109)

6. Alexey Chernov, Abstract sensitivity analysis for nonlinear equations and applications, Numerical Mathematics and Advanced Applications (Karl Kunisch, Günther Of, and Olaf Steinbach, eds.), Proceedings of ENUMATH 2007, Graz, Austria, Springer, September 2008, pp. $407-414$.

7. Alexey Chernov and Christoph Schwab, Sparse p-version BEM for first kind boundary integral equations with random loading, Appl. Numer. Math. 59 (2009), no. 11, 2698-2712. MR2566765

8. Alexey Chernov and Christoph Schwab, Sparse space-time Galerkin BEM for the nonstationary heat equation, Tech. Report 2011b10, Hausdorff Research Institute for Mathematics, University of Bonn, December 2011. 
9. Martin Costabel, Boundary integral operators for the heat equation, Integral Equations Operator Theory 13 (1990), no. 4, 498-552. MR1058085 (91j:35119)

10. Giuseppe Da Prato, An introduction to infinite-dimensional analysis, Universitext, SpringerVerlag, Berlin, 2006, Revised and extended from the 2001 original by Da Prato. MR 2244975 (2009a:46001)

11. M. Griebel and H. Harbrecht, On the construction of sparse tensor product spaces, Tech. report, 2011, submitted to Math. Comp. Also available as INS Preprint No. 1104.

12. M. Griebel and P. Oswald, Tensor product type subspace splittings and multilevel iterative methods for anisotropic problems, Adv. Comput. Math. 4 (1995), no. 1-2, 171-206. MR.1338900 (96e:65069)

13. Jacques Hadamard, Lessons on the calculus of variations (in french), 1910.

14. Helmut Harbrecht, Reinhold Schneider, and Christoph Schwab, Sparse second moment analysis for elliptic problems in stochastic domains, Numer. Math. 109 (2008), no. 3, 385-414. MR2399150 (2009b:65012)

15. Ralf Hiptmair and Jingzhi Li, Shape derivatives in differential forms I: An intrinsic perspective, Tech. Report 2011-42, ETH Zurich, 2011.

16. L. V. Kantorovich and G. P. Akilov, Funktsionalnyi analiz (Russian) [functional analysis], third ed., "Nauka", Moscow, 1984. MR788496 (86m:46001)

17. A. N. Kolmogorov and S. V. Fomin, Elementy teorii funktsii i funktsionalnogo analiza (Russian) [elements of the theory of functions and functional analysis], seventh ed., FIZMATLIT, Moscow, 2004.

18. Michel Ledoux and Michel Talagrand, Probability in Banach spaces, Ergebnisse der Mathematik und ihrer Grenzgebiete (3) [Results in Mathematics and Related Areas (3)], vol. 23, Springer-Verlag, Berlin, 1991, Isoperimetry and processes. MR1102015 (93c:60001)

19. Claudia Prévôt and Michael Röckner, A concise course on stochastic partial differential equations, Lecture Notes in Mathematics, vol. 1905, Springer, Berlin, 2007. MR2329435 (2009a:60069)

20. Stefan A. Sauter and Christoph Schwab, Boundary element methods, Springer Series in Computational Mathematics, vol. 39, Springer-Verlag, Berlin, 2011, Translated and expanded from the 2004 German original. MR2743235 (2011i:65003)

21. Christoph Schwab and Wolfgang L. Wendland, On the extraction technique in boundary integral equations, Math. Comp. 68 (1999), no. 225, 91-122. MR1620247(99d:65327)

22. Christoph Schwab and Claude Jeffrey Gittelson, Sparse tensor discretizations of highdimensional parametric and stochastic PDEs, Acta Numer. 20 (2011), 291-467. MR2805155

23. Christoph Schwab and Rob Stevenson, Space-time adaptive wavelet methods for parabolic evolution problems, Math. Comput. 78 (2009), 1293-1318. MR2501051(2011a:65347)

24. Christoph Schwab and R. A. Todor, Sparse finite elements for stochastic elliptic problemshigher order moments, Computing 71 (2003), no. 1, 43-63. MR2009650 (2004k:65226)

25. Jan Sokołowski and Jean-Paul Zolésio, Introduction to shape optimization, Springer Series in Computational Mathematics, vol. 16, Springer-Verlag, Berlin, 1992, Shape sensitivity analysis. MR.1215733 (94d:49002)

26. Tobias von Petersdorff and Christoph Schwab, Sparse finite element methods for operator equations with stochastic data, Appl. Math. 51 (2006), no. 2, 145-180. MR2212311

27. Eberhard Zeidler, Nonlinear functional analysis and its applications. I, Springer-Verlag, New York, 1986, Fixed-point theorems, Translated from the German by Peter R. Wadsack. MR816732(87f:47083)

Hausdorff Center for Mathematics \& Institute for Numerical Simulation, UniverSITY OF BONN, 53115 BONN, GERMANY

E-mail address: chernov@hcm.uni-bonn.de

Seminar for Applied Mathematics, ETH Zürich, 8092 Zürich, Switzerland

E-mail address: schwab@sam.math.ethz.ch 